\title{
Shaping of Signal Transmission at the Photoreceptor Synapse by EAAT2 Glutamate Transporters
}

\author{
Stephanie Niklaus, ${ }^{1,3, *}$ Lucia Cadetti, ${ }^{1, *}$ () Colette M. vom Berg-Maurer, ${ }^{1}$ (1) André Lehnherr, ${ }^{1,3}$ Adriana L. \\ Hotz, ${ }^{1}$ Ian C. Forster, ${ }^{2}$ Matthias Gesemann, ${ }^{1}$ and ${ }^{-S t e p h a n ~ C . F . ~ N e u h a u s s ~}{ }^{1}$
}

DOI:http://dx.doi.org/10.1523/ENEURO.0339-16.2017

${ }^{1}$ Institute of Molecular Life Sciences, University of Zurich, Zurich, CH-8057, Switzerland, ${ }^{2}$ Institute of Physiology, Zurich, CH-8057, Switzerland, and ${ }^{3}$ Life Science Zürich Graduate Program - Neuroscience, Zurich, CH-8057,

Switzerland

Visual Abstract
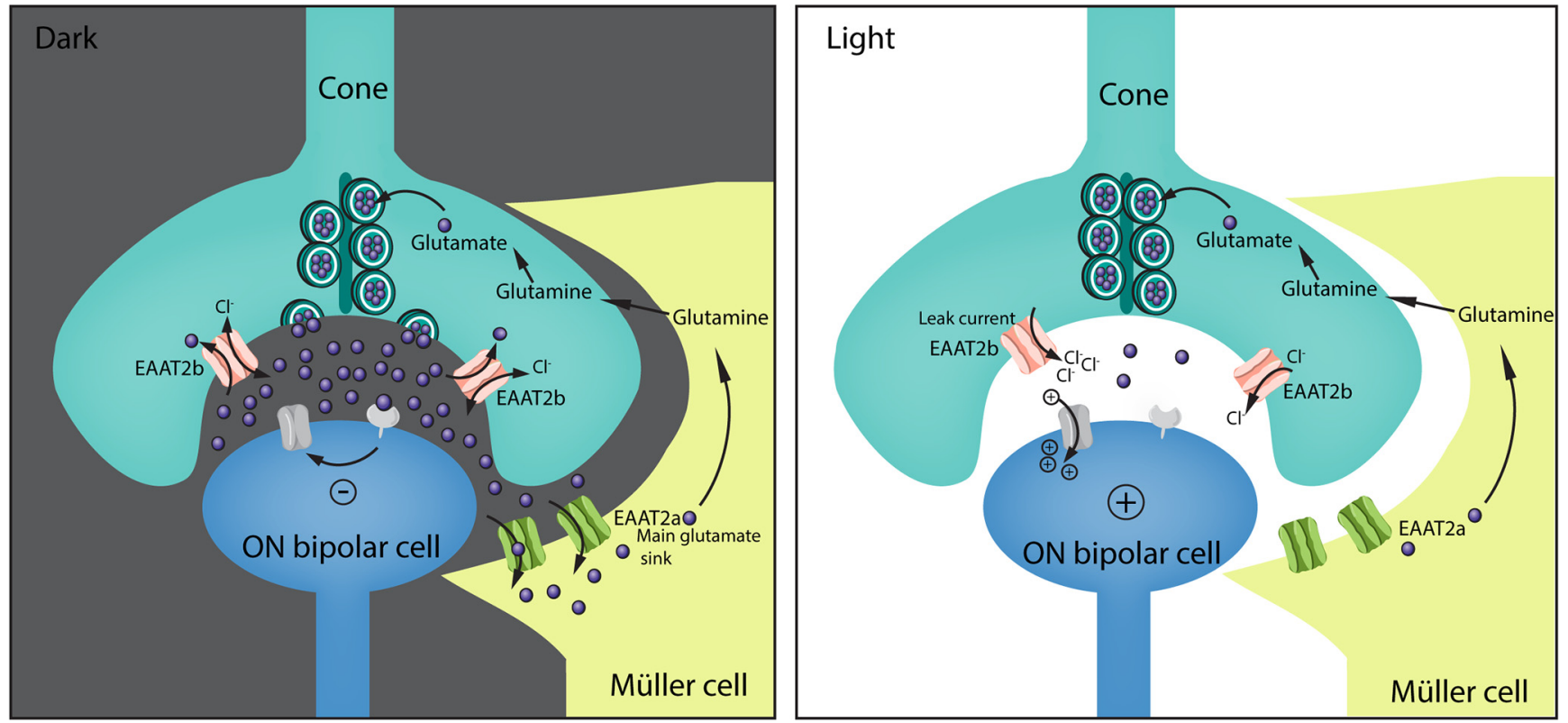

Photoreceptor ribbon synapses tonically release glutamate. To ensure efficient signal transmission and prevent glutamate toxicity, a highly efficient glutamate removal system provided by members of the SLC1 gene family is required. By using a combination of biophysical and in vivo studies, we elucidate the role of excitatory amino acid transporter 2 (EAAT2) proteins in synaptic glutamate homeostasis at the zebrafish photoreceptor synapse. The

\section{Significance Statement}

Glutamate transporters are key regulators of glutamate homeostasis. Here we analyze two players of glutamate homeostasis at the zebrafish photoreceptor synapse. This report demonstrates how paralogous glutamate transporters emerging from a whole-genome duplication event acquired a complementary expression pattern and adopted different biophysical characteristics that allow modulation of the synapse and signal transmission in a specialized manner. 
main glutamate sink is provided by the glial EAAT2a, reflected by reduced electroretinographic responses in EAAT2a-depleted larvae. EAAT2b is located on the tips of cone pedicles and contributes little to glutamate reuptake. However, this transporter displays both a large chloride conductance and leak current, being important in stabilizing the cone resting potential. This work demonstrates not only how proteins originating from the same gene family can complement each other's expression profiles and biophysical properties, but also how presynaptic and glial transporters are coordinated to ensure efficient synaptic transmission at glutamatergic synapses of the central nervous system.

Key words: Excitatory amino acid transporter; glutamate; retina; zebrafish

\section{Introduction}

Excitatory amino acid transporters (EAATs) are highaffinity glutamate transporters that regulate the extracellular concentration of glutamate in the vertebrate brain and retina. The transport of glutamate is an electrogenic process driven by the cotransport of three sodium and one hydrogen and the countertransport of one potassium ion (Zerangue and Kavanaugh, 1996). In addition, a thermodynamically uncoupled chloride conductance, whose magnitude differs between EAATs, is associated with the glutamate transport. This chloride conductance can modulate the cell's membrane potential (Picaud et al., 1995; Palmer et al., 2003; Veruki et al., 2006; Wersinger et al., 2006). Photoreceptors possess highly specialized ribbon synapses, where vesicles are tethered to provide tonic release of glutamate (Morgans, 2000; Schmitz, 2009). In contrast to nonribbon synapses, where the depolarization of the presynaptic terminal induces the release of a few vesicles of neurotransmitter, cone ribbon synapses allow the simultaneous release of hundreds of vesicles. This can result in exceptionally high glutamate concentrations within the synapse, making efficient glutamate uptake capacities essential to ensure an optimal signal-to-noise ratio, precisely terminate a signal, and prevent neurotoxic glutamate concentrations (Morgans, 2000). As a consequence, EAATs are highly expressed in the retina. In mammals, the main retinal glutamate uptake is conducted

Received November 10, 2016; accepted May 25, 2017; First published June 5, 2017.

Authors report no conflict of interest.

Authors' Contributions: SN performed histology and electroretinography, prepared Figs. 1-4, and wrote the manuscript; LC made oocyte recordings, prepared Fig. 5, and contributed to manuscript writing; CMvBM cloned the genes, did initial physiological and histological analyses, and wrote an earlier version of the manuscript; AL and ALH contributed to expression analyses; ICF contributed to oocyte recordings; MG contributed to cloning and phylogenetic analyses; SCFN conceived and coordinated the study and contributed to manuscript writing; and all authors edited and approved the manuscript.

Funding: This work was supported by the Swiss National Science Foundation (31003A_153289/1).

${ }^{*}$ S.N. and L.C. contributed equally to this work.

Colette M. vom Berg-Maurer's present address: Eawag, Swiss Federal Institute of Aquatic Science and Technology, 8600, Dübendorf, Switzerland.

Acknowledgments: We thank Kara Dannenhauer for excellent technical and animal support and Dr. Shoji Kawamura Kennedy for transgenic lines.

Correspondence should be addressed to Stephan C.F. Neuhauss, Institute of Molecular Life Sciences, University of Zurich, Winterthurerstrasse 190, CH-8057 Zurich, Switzerland. E-mail: stephan.neuhauss@imls.uzh.ch.

DOI:http://dx.doi.org/10.1523/ENEURO.0339-16.2017

Copyright $\odot 2017$ Niklaus et al.

This is an open-access article distributed under the terms of the Creative Commons Attribution 4.0 International license, which permits unrestricted use, distribution and reproduction in any medium provided that the original work is properly attributed. by Müller glia cells through EAAT1 (GLAST; White and Neal, 1976; Harada et al., 1998; Rauen et al., 1998; Barnett and Pow, 2000; Rauen, 2000; Levinger et al., 2012; Tse et al., 2014). However, in most other vertebrates including zebrafish (Danio rerio), the glutamate transporter expressed on Müller glia cells is EAAT2 (GLT-1; Eliasof et al., 1998a, b). Interestingly, EAATs are also present at photoreceptor terminals, where glutamate uptake is accompanied by a subtle regulation of the presynaptic potential (Eliasof and Werblin, 1993; Vandenbranden et al., 1996; Gaal et al., 1998; Roska et al., 1998; Hasegawa et al., 2006; Rowan et al., 2010; Szmajda and Devries, 2011).

In the present study, we focus on the role of EAAT2 at the photoreceptor synapse of the cone-dominant zebrafish retina. The zebrafish genome harbors two eaat2 genes that originated from a whole-genome duplication event $\sim 350$ million years ago (Gesemann et al., 2010b). For EAAT2, we found an unusual case of both spatial and functional subfunctionalization (division of the ancestral gene function among the two paralogs, as reviewed by Glasauer and Neuhauss [2014]). Biophysical properties of the transporters differ in dependence of their biological requirements. EAAT2a is expressed in Müller glia cells, and knockdown of this glial transporter significantly reduces the electroretinogram (ERG) b-wave amplitude, indicating that EAAT2a removes the main load of synaptic glutamate. EAAT2b is located presynaptically in cone pedicles. Loss of EAAT2b does not significantly reduce the ERG b-wave, suggesting that it interferes only slightly with the removal of cleft glutamate: its action could be limited to the cone presynaptic terminal in agreement with its proposed function of accelerating the transient cone response (Rowan et al., 2010). Furthermore, with its high chloride conductance and the presence of a significant leak current, EAAT2b may stabilize the dark resting potential of cones. This presents an intriguing case of divergent evolution of an ancestral protein leading to subfunctionalized proteins at the photoreceptor synapse, supporting vision through different mechanisms.

\section{Methods}

\section{Fish maintenance}

Zebrafish (Danio rerio) of the Tübingen and Wik strain were kept in a 14-h/10-h light/dark cycle under standard conditions as previously described (Mullins et al., 1994). Larval stages were raised in E3 embryo medium $(5 \mathrm{mM}$ $\mathrm{NaCl}, 0.17 \mathrm{~mm} \mathrm{KCl}, 0.33 \mathrm{~mm} \mathrm{CaCl}, 0.33 \mathrm{~mm} \mathrm{MgSO}_{4}$, $10^{-5} \%$ methylene blue) at $28^{\circ} \mathrm{C}$. All experiments were performed in accordance with the ARVO Statement for 
the Use of Animals in Ophthalmic and Vision Research and were approved by the local authorities (Veterinäramt Zürich TV4206).

\section{In situ hybridization}

Cloning of the eaat2 genes into the TOPO pCRII vector (TA Cloning Kit Dual Promoter, Invitrogen) is described elsewhere (Gesemann et al., 2010a). Plasmids containing the genes were linearized for SP6 and T7 in vitro transcription and purified with phenol-chloroform. Digoxigenin (DIG)-labeled antisense RNA probes were generated using DIG-RNA-labeling kit (Roche Diagnostics). Larval whole-mount and adult retina in situ hybridization was done on 5-d postfertilization (5-dpf) larvae and adult retinal cross sections. Detailed protocol of in situ hybridization is described by Huang et al. (2012). Briefly, the tissue was treated with proteinase $\mathrm{K}$ and postfixed with $4 \%$ paraformaldehyde (PFA) before prehybridization at $64^{\circ} \mathrm{C}$. Hybridization of RNA probes was done at $64^{\circ} \mathrm{C}$ overnight. On day 2 , after several stringency washes at $64^{\circ} \mathrm{C}$, probes were blocked in $1 \times$ Roche blocking solution in Tris/ $\mathrm{NaCl} /$ Tween. Anti-DIG AP antibody was applied overnight at $4^{\circ} \mathrm{C}$. On day 3 , after several washing steps, signal was detected by incubation in staining buffer. Stained embryos/retinal sections were fixed with PFA and imaged in glycerol (whole-mount) with an Olympus BX61 light microscope. Images were processed and assembled using Adobe Photoshop and Adobe Illustrator CS5.

\section{Generation of antibodies}

Chickens were immunized using the peptide $\mathrm{H}_{2} \mathrm{~N}-\mathrm{CKL}$ KEN LGE GLE NDE V-CONH${ }_{2}$ to raise chicken antiEAAT2a antibodies. Antibodies against EAAT2b were raised in guinea pigs using the peptides $\mathrm{H}_{2} \mathrm{~N}-\mathrm{CKL}$ KAN LGE GKK NDE V-CONH$H_{2}$ and $\mathrm{H}_{2} \mathrm{~N}-\mathrm{CKG}$ AAK YVI KKS $\mathrm{LQF} \mathrm{KS}-\mathrm{CONH}_{2}$. Antibodies were raised in a 87-d classic program and affinity-purified against the corresponding peptides by Eurogentec (Seraing, Belgium).

\section{Immunohistochemistry}

Larvae (5 dpf) or adult eyes were fixed in 4\% PFA in PBS, $\mathrm{pH} 7.4$, or $2 \%$ trichloroacetic acid for 30 min at room temperature. Samples were cryoprotected in 30\% sucrose in PBS overnight at $4^{\circ} \mathrm{C}$ and embedded in cryomatrix (Tissue Tek OCT Compound, Sakura Finetek) using liquid $\mathrm{N}_{2}$ to immediately freeze the samples. Sections of 16-18 $\mu \mathrm{m}$ were mounted onto Superfrost slides (Thermo Fisher Scientific). Slides were air-dried at room temperature and stored at $-20^{\circ} \mathrm{C}$. Before use, slides were thawed at $37^{\circ} \mathrm{C}$ for $30 \mathrm{~min}$ and washed in PBS, $\mathrm{pH} 7.4$, for $10 \mathrm{~min}$. Blocking solution (10\% normal goat serum, 1\% bovine serum albumin, $0.3 \%$ Tween 20 in PBS, $\mathrm{pH}$ 7.4) was applied for at least $45 \mathrm{~min}$ at room temperature, and primary antibodies diluted in blocking solution were incubated overnight at $4^{\circ} \mathrm{C}$. The following antibodies were used: mouse anti-glutamine synthetase (EMD Millipore, MAB302) 1:700, mouse anti-Zpr-1 (Fret43, a commonly used marker labeling red-green double cones; Larison and Bremiller, 1990; Zebrafish International Resource Center) 1:400, chicken anti-EAAT2a 1:100, and guinea pig anti-EAAT2b 1:100. The immunoreaction was then de- tected using fluorescently labeled secondary antibodies (goat anti-mouse Alexa Fluor 488 or 568, goat anti-guinea pig Alexa Fluor 488 or 568, all from Invitrogen, and rabbit anti-chicken IgY Cy5 from Jackson ImmunoResearch or rabbit anti-chicken Alexa Fluor 488 from Invitrogen) diluted 1:1000 in PBS. Bodipy TR Methyl Ester (Invitrogen) was used to counterstain green fluorescence. It was applied 1:300 in PDT (PBS with 1\% DMSO and 0.1\% Triton) for 20 min after washing the secondary antibodies.

Slides were coverslipped and imaged with a SP5 and a TCS LSI confocal microscope (both Leica Microsystems). Images were then processed with Imaris (Bitplane) and postprocessed using Gimp imaging processing software, Adobe Photoshop, and Adobe Illustrator CS5.

\section{Histology}

Whole larvae were fixed in $4 \%$ PFA overnight at $4^{\circ} \mathrm{C}$. Larvae were dehydrated in series of increasing ethanol concentrations in PBS (50\%, 70\%, 80\%, 90\%, 95\%, and $100 \%$ ethanol). After dehydration, larvae were incubated in a 1:1 ethanol Technovit 7100 (Heraeus Kulzer) solution (1\% Hardener 1 in Technovit 7100 basic solution) for $1 \mathrm{~h}$ followed by incubation in $100 \%$ Technovit solution overnight at room temperature. Larvae were then embedded in plastic molds in Technovit 7100 polymerization medium and dried at $37^{\circ} \mathrm{C}$ for $1 \mathrm{~h} .3-\mu \mathrm{m}$-thick sections were prepared with a microtome, mounted onto slides, and dried at $60^{\circ} \mathrm{C}$. Sections were stained with Richardson-Romeis $(0.5 \%$ borax, 0.5\% Azur II, 0.5\% methylene blue), and slides were mounted with Entellan (Merck). Images were taken in the bright-field mode of a BX61 microscope (Olympus).

\section{Gene knockdown}

Two different EAAT2a translation-blocking morpholinos were used for injections into the one-cell stage of the zebrafish embryo. Titration injections were performed to find the optimal dose with highest knockdown efficiency but no toxic side effects. For EAAT2a morpholino 1, CATCATCCACAACTGTCAGGCTGGC (position -22 to -03 ), the injected amount was $1.3 \mathrm{ng}$. For EAAT2a morpholino 2, CGTGCTTCGGCATCATCCACAACTG (position -12 to -13), two different amounts were injected, 1.8 and $3.6 \mathrm{ng}$ (referred to as low and high dose, respectively). EAAT2b morpholino 1, GATCTCCACTTGCTTCTGCATCTTC (position -04 to -21 ), was injected at a dose of $1.8 \mathrm{ng}$, and morpholino 2, GAGTITCACAACAGTTTGCTAGACA (position -65 to -41 ), was injected at a dose of $9 \mathrm{ng}$.

Before dilution, stock morpholinos were heated to $65^{\circ} \mathrm{C}$ for 5 min and subsequently diluted with nuclease free $\mathrm{H}_{2} \mathrm{O}$ to the desired concentration (injection mix contained $0.04 \%$ phenol red for color indication). The injection amount was $1 \mathrm{nl}$. The standard control morpholino was injected into sibling embryos. Injected embryos were raised in E3 containing methylene blue at $28^{\circ} \mathrm{C}$ until $5 \mathrm{dpf}$.

Efficiency of knockdown was quantified by measuring the fluorescence of antibody staining on wild-type (WT) and morphant retinal sections (imaged with the same confocal microscope settings). For EAAT2a, a region of interest $(\mathrm{ROI})$ reaching from photoreceptors to basal processes of Müller cells was selected to measure the fluorescence. For EAAT2b, the ROI was placed over an area 
of the outer plexiform layer (OPL). Because EAAT2b staining is present only in OPL, background fluorescence [ROI set on inner nuclear layer (INL)] was subtracted from fluorescence in OPL. Two ROls were set per animal. One-way ANOVA with Tukey post hoc test was performed to statistically analyze the difference in fluorescence.

\section{Assessment of retinal morphology in WT and EAAT2 knockdown animals}

Retinal morphology of EAAT2a and EAAT2b morphant and WT animals was analyzed on cryosections, and several parameters were statistically compared. 5-dpf WT $(n=13)$ and larvae injected with EAAT2a MO 1 (1.3 ng; $n=13)$, EAAT2a MO 2 ( $n=12)$, EAAT2b MO 1 (1.8 ng; $n=$ 13), and EAAT2b MO 2 (9 ng; $n=13$ ) were fixed, embedded in cryomatrix, and sectioned at $16 \mu \mathrm{m}$ before immunofluorescent staining with Zpr-1 (EAAT2b morphants) and glutamine synthetase (EAAT2a morphants; see Materials and Methods, "Immunohistochemistry"). The retinal thickness was measured on confocal images using Fiji software. Retinal thickness was assessed on three different locations per larva. The average of the three measurements was used for statistical analysis. The length of Müller cells (in EAAT2a morphants) as well as the overall cone length (EAAT2b morphants) was assessed the same way. In total, three cones and three Müller cells per larvae were analyzed (same sample sizes as for retinal thickness analysis), and the average of the three measurements was used for statistical analysis. One-way ANOVA followed by Tukey post hoc test was performed using SPSS.

\section{Electroretinography}

ERG recordings were performed on 5-d-old morphant larvae of either sex and their control injected siblings as previously described by Makhankov et al. (2004) with some minor adaptations. Larvae were dark-adapted for at least $30 \mathrm{~min}$, and all preparations before the actual recording were done under a red light, preventing bleaching of photoreceptors. The larval eye was removed from the eyecup and placed onto filter paper on top of a $1.5 \%$ agarose gel in E3, in which the reference electrode was inserted. The recording electrode (micropipette filled with E3) was placed onto the center of the cornea. 100-ms light stimuli of five different intensities (log-4 to $\log 0$ ) were given with an interstimulus interval of $7 \mathrm{~s}$. The light intensity of log0 corresponds to 6800 lux or $75 \mathrm{~W} / \mathrm{m}^{2}$.

b-wave amplitudes and the time from light onset to the b-wave peak were compared using mixed repeatedmeasures ANOVA followed by Tukey and Games-Howell post hoc test. Results are shown in a box-and-whisker plot (b-wave amplitudes and time from light onset to peak), where the bottom and top of the box represent the first and third quartile, respectively, with the median represented as a line within the box and whiskers reaching to minimum and maximum values obtained.

\section{Oocyte experiments}

Xenopus oocytes were purchased from Ecocyte Bioscience or were obtained from the Institute of Physiology, University of Zurich. The oocytes were kept at $16^{\circ} \mathrm{C}$ for 2-5 d. Capped cRNA was microinjected into Xenopus oocytes (40-80 ng per oocyte), and membrane currents were recorded 2-4 d later.

With the exception of the chloride-free solution, the extracellular recording solution comprised (in $\mathrm{mM}$ ) 96 $\mathrm{NaCl}, 2 \mathrm{KCl}, 1.8 \mathrm{CaCl}_{2}, 1 \mathrm{MgCl}_{2}$, and 5 HEPES, pH 7.5. L-Glutamate was obtained from Sigma; DL-TBOA (DLthreo- $\beta$-benzyloxyaspartic acid) was obtained from Tocris. For chloride-free solutions, all chloride salts were replaced with gluconate salts. To increase the uncoupled current, the oocytes were dialyzed in chloride-free solution for $24 \mathrm{~h}$, and the recordings were then performed in a solution in which chloride was substituted with the more permeant ion $\mathrm{SCN}^{-}$. The approximated chloride equilib-

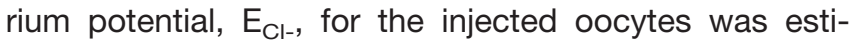
mated by calculating the reversal potential of $\mathrm{I}_{\mathrm{Cl}(\mathrm{Ca})}$ induced by 4-Br A23187 (Sigma), a calcium ionophore, as described by Wadiche et al. (1995).

Radiolabeled glutamate uptake was performed under voltage clamp at $-60 \mathrm{mV}$ (or $-15 \mathrm{mV}$ when uptake at the chloride equilibrium potential was investigated). Currents were recorded during application of $100 \mu \mathrm{M}\left[{ }^{3} \mathrm{H}\right] \mathrm{L}-$ glutamate for $100 \mathrm{~s}$. The specific activity of the $\left[{ }^{3} \mathrm{H}\right] \mathrm{L}-$ Glutamate in this solution was $20 \mathrm{Ci} / \mathrm{mol}$. For control, we used the same protocol on uninjected oocytes. After application of $\left[{ }^{3} \mathrm{H}\right] \mathrm{L}$-glutamate, oocytes were first washed in Ringer solution and then lysed in a scintillation vial containing $2 \%$ SDS. Radioactivity was later measured. Twoelectrode voltage-clamp recordings were performed using a Turbo Tex-03x amplifier (NPI), and signals were acquired with a Digidata 1440A (Molecular Devices). Recording electrodes with resistance $<1 \mathrm{M} \Omega$ contained $3 \mathrm{M}$ $\mathrm{KCl}$. Currents were acquired at $10 \mathrm{kHz}$ and low-pass filtered at 20 or $500 \mathrm{~Hz}$ in dependence of the recording protocol.

Data analysis was performed using Axon pClamp10 and GraphPad Prism software. To determine $K_{m}$, doseresponse curves were fitted with the Michaelis-Menten relationship included with the GraphPad Prism software, $Y=I_{\max } \times X /\left(K_{m}+X\right)$, where $I_{\max }$ is the maximum current in the same units as $Y$ and $K_{m}$ is the Michaelis-Menten constant, in the same unit as $X$.

\section{Results}

\section{EAAT2 paralogs are complementarily expressed in the retina}

A phylogenetic analysis of excitatory amino acid transporter genes revealed that the zebrafish genome harbors two eaat2 (also called glt-1 or s/c1a2) genes (Gesemann et al., 2010a). Analyzing the transcript expression of the two transporters in the zebrafish retina, we found strong eaat2a expression in the INL in close proximity to the inner plexiform layer (IPL) and additionally very weak expression in photoreceptors (Fig. $1 A, B$ ). In contrast, eaat $2 b$ mRNA was present in the photoreceptor cell layer and in low concentration in the INL (Fig. 1C, D). Staining in the photoreceptor layer was present throughout all cone photoreceptors; however, the layer containing nuclei of rods was unstained, suggesting eaat $2 b$ to be cone specific.

To confirm cellular identity and obtain information about subcellular distribution of the protein, we generated paralog- 

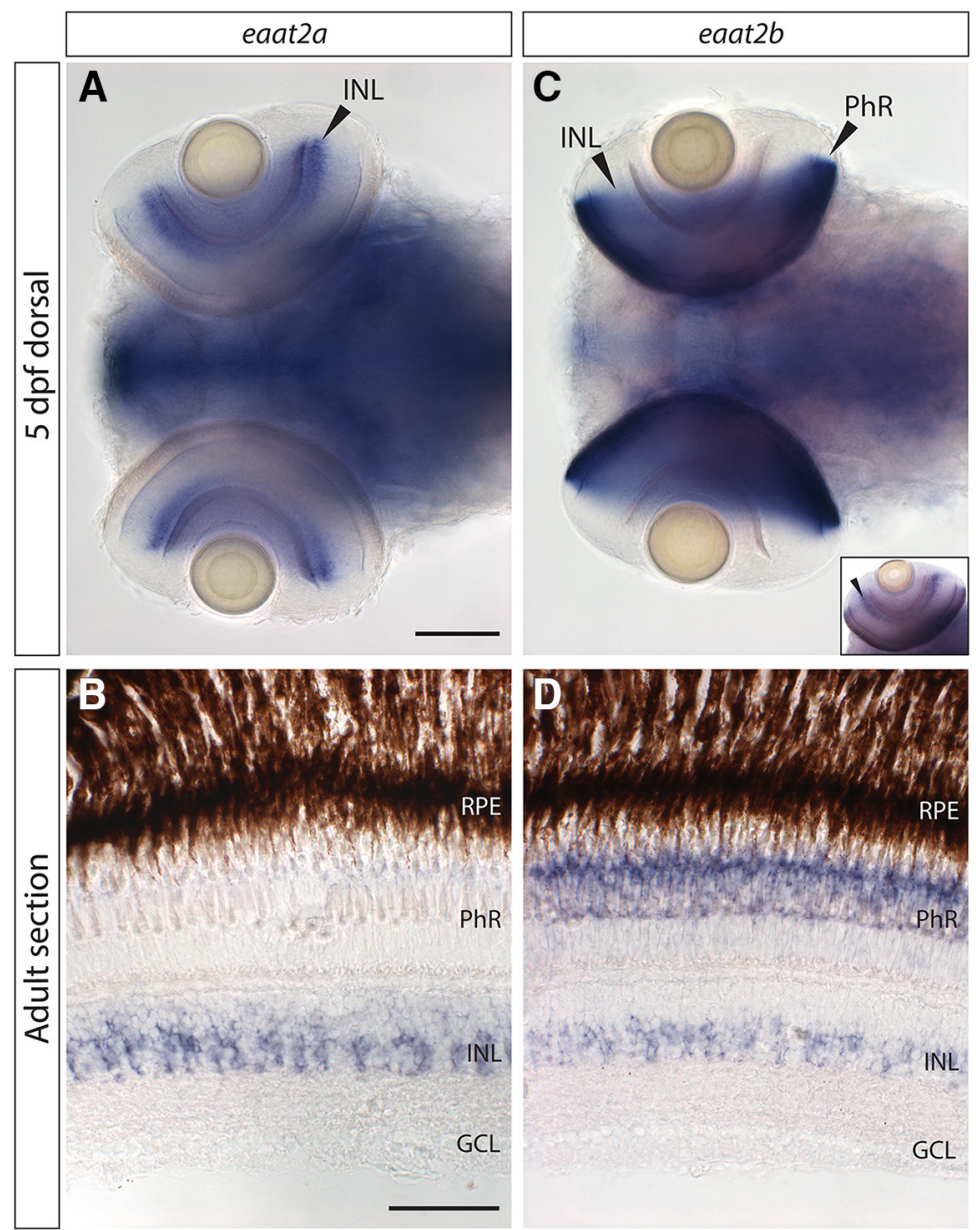

Figure 1. Transcript expression of excitatory amino acid transporter 2 (eaat2) paralogs. $\boldsymbol{A}, \boldsymbol{B}$, eaat2a mRNA is strongly expressed in the inner nuclear layer (INL) in the retina, in both 5-d postfertilization (5-dpf) larvae $(\boldsymbol{A})$ and adult retina $(\boldsymbol{B})$. Additionally, extremely low transcript levels can be found in photoreceptors $(\boldsymbol{B})$. $\boldsymbol{C}, \boldsymbol{D}, \mathrm{mRNA}$ of eaat2b is expressed in photoreceptors and weakly in the INL throughout different developmental stages ( $\boldsymbol{C}$, in 5-dpf larvae; $\boldsymbol{D}$, in adult retinal sections). Small inset in $\boldsymbol{C}$ shows eaat2b in situ staining in an eye of a whole-mount larva that has been only shortly stained, to better visualize expression in the INL. Scale bar in $\boldsymbol{A}$ is $100 \mu \mathrm{m}$; also applies to $\boldsymbol{C}$. Scale bar in $\boldsymbol{B}$ corresponds to $50 \mu \mathrm{m}$; also applies to $\boldsymbol{D}$.

specific peptide antibodies. Consistent with the in situ hybridization data, immunostainings against EAAT2a on larval and adult retinal sections revealed expression of EAAT2a in Müller glia cell membranes (Fig. 2A-F). EAAT2a seems to be specific to Müller cells, as no immunofluorescent signal could be detected in photoreceptors. The staining intensity within Müller glia cells appeared increased in the OPL and IPL, where glutamatergic synapses are tightly ensheathed by Müller glia cells. The nature of the labeled cells was confirmed by double-labeling with the glial marker glutamine synthetase, which colocalizes with EAAT2a (Fig. 2A,D). Consistent with the mRNA distribution, we found dotted antibody staining against EAAT2b protein in the OPL (Fig. $2 G-N$ ). The appearance of the staining indicated localization of EAAT2b in cone pedicles, whereas rod spherules were devoid of EAAT2b protein. To further characterize the photoreceptor subtype-specific expression, we used transgenic fish lines expressing GFP in different photoreceptor cell types [Tg(zfSWS1-5.5A:EGFP), Tg(zfSWS2-3.5A:EGFP), Tg(zfRh1-3:EGFP); Hamaoka et al., 2002; Takechi et al., $2003,2008]$. EAAT2b is expressed in the presynaptic termi- nal of all cone subtypes (UV-, blue-, red-, and greensensitive cones; Fig. 2G-I, K-M); however, consistent with the in situ hybridization results, rod terminals do not express this transporter (Fig. 2J). Remarkably, no EAAT2b immunofluorescence could be observed in cells of the INL, suggesting that local protein concentrations in the membranes of these cells are rather low, preventing immunostaining.

\section{EAAT2 paralogs differentially shape signal transmission in the cone synapse}

To characterize the function of EAAT2 transporters in zebrafish vision, we generated knockdown larvae using different morpholino antisense oligonucleotides. Quantification of fluorescence intensities in morphant and WT eyes demonstrated the efficiency of protein knockdown in 5-dpf larvae (Fig. 3).

Both EAAT2a- and EAAT2b-deficient animals showed no apparent overall morphologic abnormalities (Fig. $4 A-C, G-l)$ or abnormalities in the cell shape of Müller glia cells (Fig. $4 D-F$ ) or red and green cones (Fig. $4 J-L$ ). Statistical analysis confirmed this assessment, as no sig- 



Figure 2. Protein expression of EAAT2 paralogs. $\boldsymbol{A}-\boldsymbol{F}$, Double immunostaining of EAAT2a (green) and glutamine synthetase (magenta) in adult $(\boldsymbol{A})$ and larval $(5 \mathrm{dpf} ; \boldsymbol{D})$ retinal sections confirm expression of EAAT2a in Müller glia cells. Separated channels are shown in $\boldsymbol{B}$ (adult), $\boldsymbol{E}$ (5 dpf; EAAT2a, green channel only), $\boldsymbol{C}$ (adult), and $\boldsymbol{F}$ (5 dpf; glutamine synthetase, magenta channel only). Scale bar in $\boldsymbol{A}$ is $30 \mu \mathrm{m}$; also applies to $\boldsymbol{B}$ and $\boldsymbol{C}$. Scale bar in $\boldsymbol{D}$ is $50 \mu \mathrm{m}$; also applies to $\boldsymbol{E}$ and $\boldsymbol{F}$. $\boldsymbol{G}-\boldsymbol{N}$, EAAT2b protein is expressed in a dotted manner in the outer plexiform layer (OPL) in all cone pedicles, but it is not expressed in rods. EAAT2b antibody staining (magenta) on adult retinal sections stained with Zpr-1 (red-green double cones, G) and on retinal sections of zebrafish expressing GFP in blue cones $(\boldsymbol{H})$, UV cones $(\boldsymbol{I})$, and rods $(\boldsymbol{J})$ confirms that EAAT2b is cone specific and is spared from rod spherules. $\boldsymbol{K}-\boldsymbol{M}$ show zoom-ins of the cone pedicles expressing EAAT2b (magenta) in red-green double cones $(\boldsymbol{K})$, blue cones $(\boldsymbol{L})$, and UV cones $(\boldsymbol{M})$. $\boldsymbol{N}$ shows larval $(5 \mathrm{dpf})$ expression of EAAT2b in magenta together with a nuclear counterstain (DAPI, blue). Scale bars in $\mathbf{G}-\boldsymbol{J}$ are $7 \mu \mathrm{m}$. Scale bars in $\mathbf{K}-\mathbf{M}$ are 2 $\mu \mathrm{m}$. Scale bar in $\boldsymbol{N}$ is $30 \mu \mathrm{m}$. 

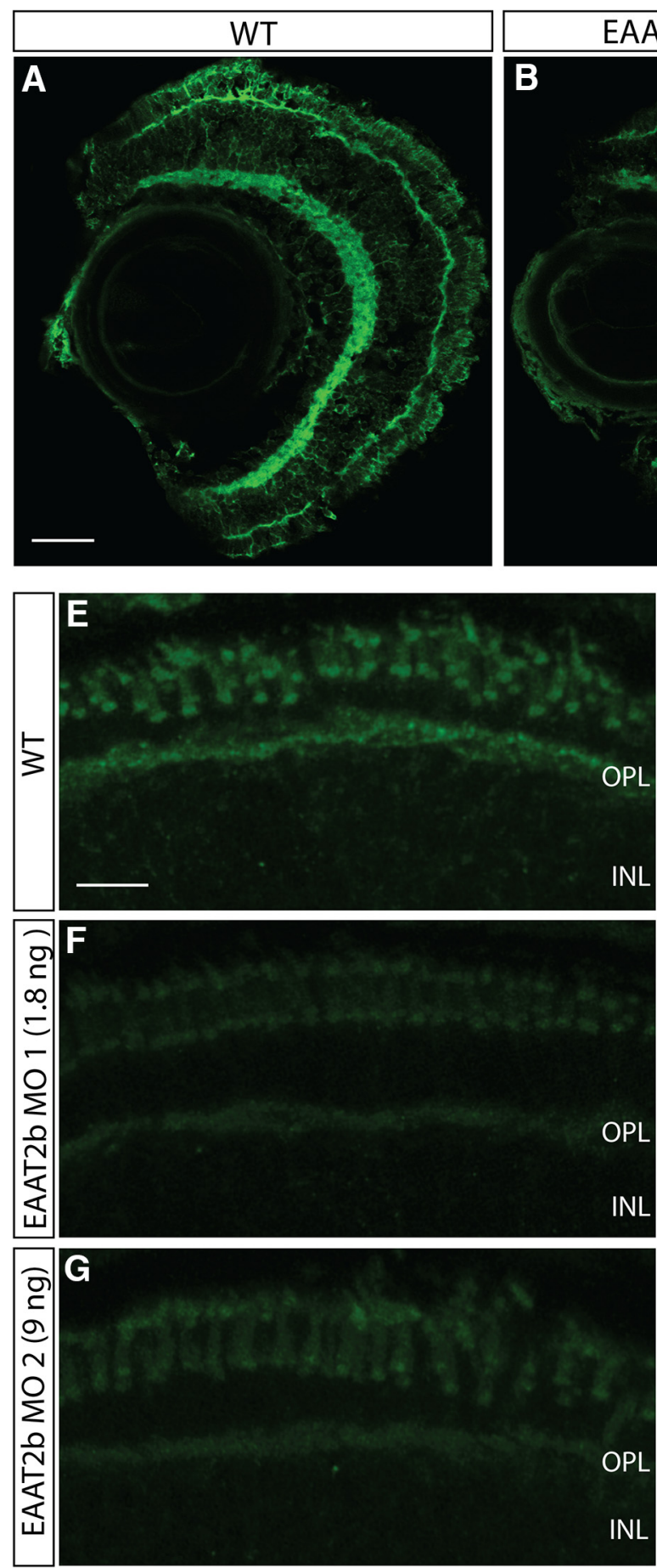
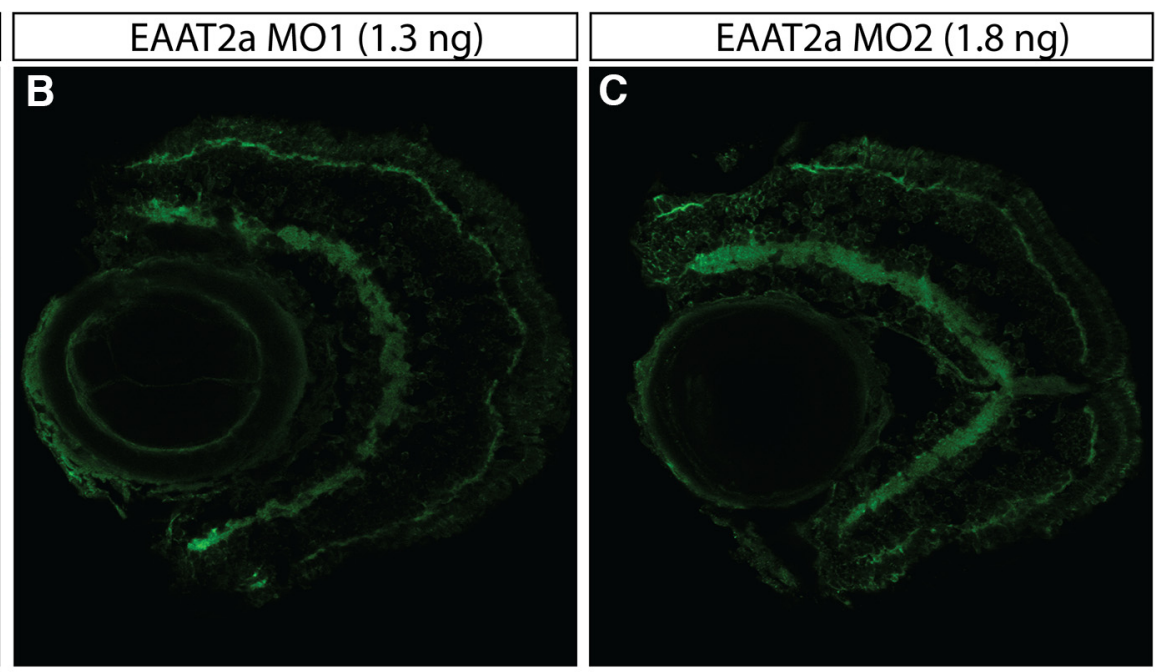

300000
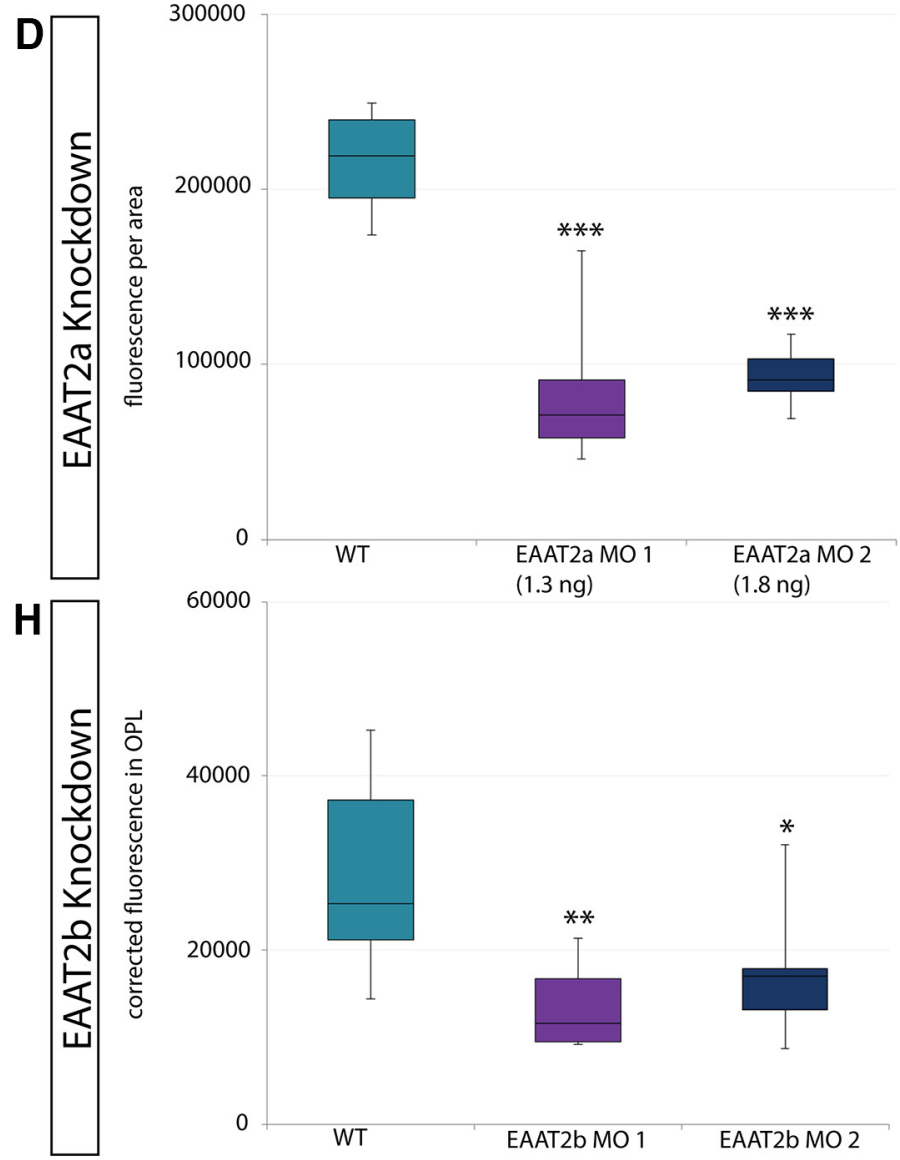

60000

(1.3 ng)

$(1.8 \mathrm{ng})$

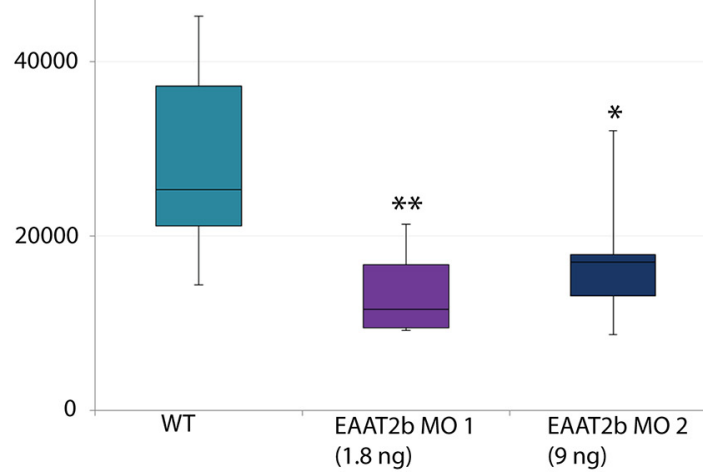

Figure 3. Confirmation of knockdown. $\boldsymbol{A}-\boldsymbol{C}$, Immunostaining of EAAT2a on WT $(\boldsymbol{A})$ and EAAT2a morphant [B, 1.3 ng EAAT2a morpholino (MO) 1; C, $1.8 \mathrm{ng}$ EAAT2a MO 2] retinal sections (5 dpf). $\boldsymbol{D}$, Box-and-whisker plot of analysis of fluorescence of WT, EAAT2a MO 1, and EAAT2a MO 2 injected animals stained with anti-EAAT2a antibody. Statistical analysis reveals a highly significant $(p<0.001)$ reduction of fluorescence for both MOs. $\boldsymbol{E}-\boldsymbol{G}$, Retinal sections of WT $(\boldsymbol{E})$ and EAAT2b morphant $(\boldsymbol{F}, 1.8 \mathrm{ng}$ EAAT2b MO 1; $\boldsymbol{G}, 9 \mathrm{ng}$ EAAT2b MO 2) larvae stained with anti-EAAT2b antibody. $\boldsymbol{H}$, Fluorescence was measured in the OPL, and background fluorescence (taken from area in INL) was subtracted. Fluorescence of WT and morphant immunostaining is plotted in a box-andwhisker plot and shows a significant $(p<0.01)$ and slightly significant $(p<0.05)$ decrease in fluorescence in animals injected with 1.8 ng EAAT2b MO 1 and 9 ng EAAT2b MO 2, respectively. EAAT2a WT, $n=6$; EAAT2a MO 1, $n=8$; EAAT2a MO 2, $n=8$; EAAT2b WT, $n=10$; EAAT2b MO 1, $n=10$; EAAT2b MO 2, $n=10$. Scale bar in $\boldsymbol{A}$ is $30 \mu \mathrm{m}$; also applies to $\boldsymbol{B}$ and $\boldsymbol{C}$. Scale bar in $\boldsymbol{E}$ is 10 $\mu \mathrm{m}$; also applies to $\boldsymbol{F}$ and $\boldsymbol{G}$. 



Figure 4. Retinal histology of EAAT2 morphant zebrafish larvae. Histologic analysis of retinal sections of WT, EAAT2a, and EAAT2b morphant zebrafish larvae (5 dpf) stained with Richardson-Romeis (A-C and $\mathbf{G}-\boldsymbol{I})$. Immunostaining of glutamine synthetase (green) labeling Müller glia cells counterstained with Bodipy (magenta; $\boldsymbol{D}-\boldsymbol{F}$ ) of WT and EAAT2a morphant (E, EAAT2a MO 1; $\boldsymbol{F}$, EAAT2a MO 2) 5-dpf retinal sections. Anti-Zpr-1 immunostaining (labeling red and green cones, shown in green) on WT (J) and EAAT2b morphant ( $\boldsymbol{K}$, EAAT2b MO 1; $\boldsymbol{L}$, EAAT2b MO 2) retinal sections counterstained with Bodipy (magenta). Knockdown of neither EAAT2a (B, EAAT2a MO 1; $\boldsymbol{C}$, EAAT2a MO 2) nor EAAT2b ( $\boldsymbol{H}$, EAAT2b MO 1; $\boldsymbol{l}$, EAAT2b MO 2) causes any defect in retinal lamination. Thickness of the retina was assessed on WT and morphant larvae and did not reveal any significant difference in the thickness of the retina in either EAAT2a or EAAT2b morphants $(\boldsymbol{M}, \mathbf{O})$, yielding $p$ values of 0.997 and 0.935 for EAAT2a MO 1 and EAAT2a MO 2, respectively, and 0.658 and 0.922 for EAAT2b MO 1 and EAAT2b MO 2 (all in comparison to WT). Moreover, knockdown of EAAT2a does not significantly influence Müller glia cell length 
continued

$(\boldsymbol{N})$, nor does the loss of EAAT2b result in cone length alteration $(\boldsymbol{P})$. Statistical analysis of the cell length yielded $p$ values of 0.969 and 0.989 for EAAT2a MO 1 and EAAT2a MO 2, respectively, and 0.911 and 0.631 for EAAT2b MO 1 and EAAT2b MO 2 (in comparison to WT). All scale bars are $50 \mu \mathrm{m}$. Scale bar in $\boldsymbol{A}$ also applies to $\boldsymbol{B}$ and $\boldsymbol{C}$; scale bar in $\boldsymbol{D}$ also applies to $\boldsymbol{E}$ and $\boldsymbol{F}$; scale bar in $\boldsymbol{G}$ also applies to $\boldsymbol{H}$ and $\boldsymbol{I}$; and scale bar in $\boldsymbol{J}$ also applies to $\boldsymbol{K}$ and $\boldsymbol{L}$.

nificant difference in retinal thickness between WT and EAAT2a (Fig. 4M) or EAAT2b morphant larvae (Fig. 4O) could be detected. Furthermore, neither the length of Müller cells (Fig. 4N) nor that of cones (Fig. 4P) was influenced by knockdown of EAAT2a and EAAT2b, respectively, indicating that the knockdown does not impair retinal development. However, EAAT2a morphant larvae displayed behavioral changes (no quantifications done) and abnormal body bends, similar to the ones described in tht mutants that carry a point mutation in eaat2a (McKeown et al., 2012).

To analyze the functional role of EAAT2 in the zebrafish retina, we performed ERG on 5-d-old morphant larvae. The ERG measures the sum field potential generated by retinal cells on light exposure. We used the amplitude of the b-wave at varying light intensities as a functional readout of outer retinal function. The b-wave reflects the activation of ON bipolar cells after photoreceptor hyperpolarization after a light stimulus. In a typical WT ERG, the large b-wave masks the a-wave, resulting in no or only a tiny a-wave visible. At $5 \mathrm{~d}$, rods are not yet functionally integrated into the larval retina; therefore, the measured light responses are exclusively cone driven (Branchek, 1984; Branchek and Bremiller, 1984). To analyze the ERG kinetics, the time from light stimulus onset to the peak of the $b$-wave was compared between control and morphant animals.

Loss of EAAT2a leads to a reduction of the ON response. EAAT2a-deficient larvae showed a highly significant reduction in b-wave amplitude throughout all different light intensities tested, indicating that this glial transporter is fundamental for uptake of the synaptic glutamate (Fig. 5A). b-wave amplitudes of larvae injected with $1.3 \mathrm{ng}$ morpholino 1 or low-dose morpholino 2 were diminished by $>40 \%$ throughout all different light intensity flashes. b-wave amplitudes of larvae injected with high-dose morpholino 2 showed a reduction of $75 \%$ or more in comparison to WT and control injected larvae. Furthermore, we show a clear dose dependence of morpholino 2, leading to significant differences in the b-wave amplitude between low- and high-dose EAAT2a morphants. This was observed for all light intensities except the lowest intensity stimulus (log-4). However, even at high doses of injected EAAT2a morpholino, we could still observe a small ERG $b$-wave, indicating that there is either no complete protein knockdown or that different glutamate transporters present at the first visual synapse take over a comparable function.

In contrast to EAAT2a, knockdown of EAAT2b resulted in only a mild ERG phenotype. The medians of ERG $b$-wave amplitudes of EAAT2b morphant larvae were slightly reduced compared with WT and control morphant ERG, but were statistically significant only for some light intensities (see legend, Fig. 5B).
To artificially augment glutamate concentrations in the cleft and disrupt the glutamate-glutamine cycle between photoreceptors and Müller glia cells, double injections of $1.3 \mathrm{ng}$ EAAT2a and $9 \mathrm{ng}$ EAAT2b morpholino were performed. Interestingly, except for dim light conditions, b-wave amplitudes of double-knockdown larvae were significantly lower than those of EAAT2a morphants (Fig. $5 C$ ). This nicely demonstrates the function of EAAT2b by illustrating that glutamate clearing capacity has further been neutralized in the EAAT2 double morphants.

Knockdown of EAAT2a affected not only the b-wave amplitude, but also its kinetics. In general, the ERG response in EAAT2a-depleted animals was slower (representative ERG traces shown in Fig. $5 D$ ) than in WT or control animals (Fig. 5E). The time to peak at low light intensity stimuli (log-4) was significantly increased in EAAT2a-depleted larvae. Also at bright light $(\log 0)$, the ERG response was still decelerated, resulting in a significant increase in the time to peak in EAAT2a-depleted animals (Fig. $6 A, B$ ). Because ON bipolar cell activity is decreased and delayed in EAAT2a morphants, the $b$-wave no longer dominates the a-wave. Therefore, the a-wave (electronegative wave preceding the positive b-wave) becomes apparent in an EAAT2a morphant ERG (Fig. 5E). ERG kinetics in EAAT2b-depleted larvae is unaffected (Fig. 6C, D; representative ERG traces are shown in Fig. 5F).

\section{Biophysical properties of EAAT2 paralogs}

Glutamate uptake by EAAT proteins is electrogenic, being associated with the movement of charges through the cell membrane: three $\mathrm{Na}^{+}$ions and one proton enter the cell for each transport cycle, and one $\mathrm{K}^{+}$ion moves out (Zerangue and Kavanaugh, 1996; Levy et al., 1998; Owe et al., 2006). In addition, on binding of glutamate and sodium, a thermodynamically uncoupled chloride current is associated with these transporters (Fairman et al., 1995; Wadiche and Kavanaugh, 1998). These intrinsic electrogenic properties of glutamate transporters allow evaluation of their electrical properties by expressing them in Xenopus oocytes.

Approximately $48 \mathrm{~h}$ after microinjection of eaat2a or eaat $2 b$ mRNA, Xenopus oocytes displayed glutamateevoked currents $\left(I_{\mathrm{Glu}}\right)$ that were absent in uninjected oocytes. In oocytes voltage-clamped to negative potentials, increasing doses of glutamate evoked an increasing inward current (Fig. 7A, $B$, insets). The normalized amplitude of $\mathrm{I}_{\mathrm{Glu}}$ for different glutamate concentrations was fitted with the Michaelis-Menten equation (Fig. $7 A, B$ ) to yield a $K_{m}$ of $19.6 \pm 2.7 \mu \mathrm{M}(n=6)$ for EAAT2a and 3.1 \pm $0.6 \mu \mathrm{M}(n=8)$ for EAAT2b (see Materials and Methods). When voltage-clamping the oocytes to different potentials and measuring $I_{\text {Glu }}$ in the steady state, we found that at sufficiently positive potentials, $I_{\text {Glu }}$ reversed for both 

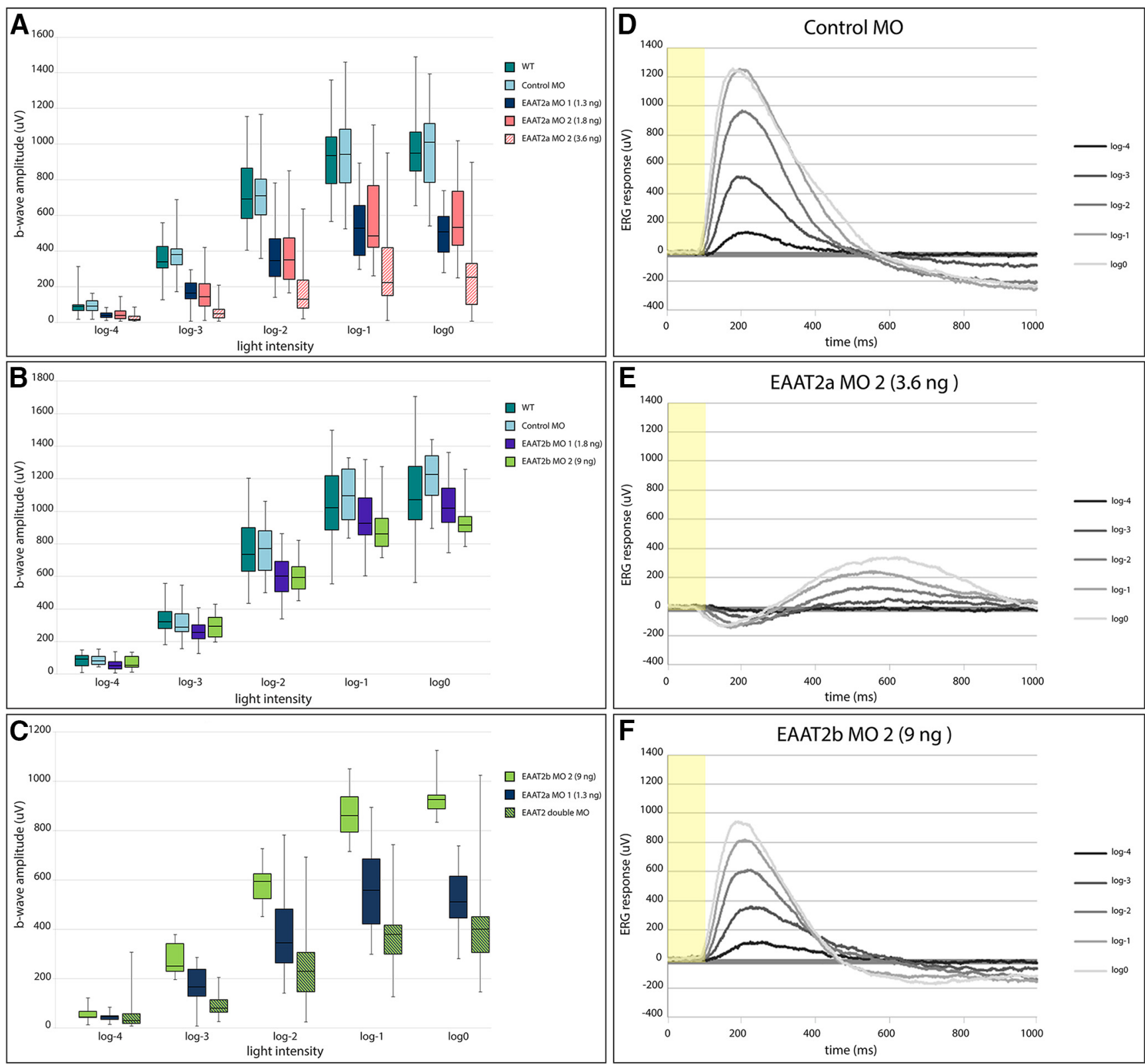

Figure 5. Box-and-whisker plots of ERG b-wave amplitudes of EAAT2a and EAAT2b morphant zebrafish larvae and representative ERG traces. $\boldsymbol{A}$, Knockdown of EAAT2a results in a highly significant $(\boldsymbol{p}<0.001)$ reduction of the ERG $b$-wave amplitude in comparison to both WT and control injected animals throughout all light intensities (log-4 to log0). For EAAT2a MO 2, we could demonstrate a dose dependence, resulting in a highly significant difference $(p<0.001)$ between the low dose $(1.8 \mathrm{ng})$ and the high dose $(3.6 \mathrm{ng})$ for the bright light intensities (log-1 and log0) and a significant difference $(p<0.01)$ for the medium light intensities (log-3 and log-2). WT, $n=33$; control MO, $n=25$; EAAT2a MO 1, $n=23 ; 1.8 \mathrm{ng}$ EAAT2a MO 2, $n=27$; $3.6 \mathrm{ng}$ EAAT2a MO 2, $n=24$. $\boldsymbol{B}$, Knockdown of EAAT2b only mildly interferes with the ERG $b$-wave. There is an overall tendency in EAAT2b-depleted animals to have a slightly reduced ERG $b$-wave amplitude. This results in a slight statistical significance $(p<0.05)$ between WT and EAAT2b morphant (MO 1$)$ at $\log -4$ and a highly significant $(p<0.001)$ reduction at log-3 and log-2. Further, there is a slightly significant $(p<0.05)$ reduction between EAAT2b morphants (MO 1) and control morphants for log-2. When using EAAT2b MO 2, we obtained a significant $(p<0.01$ ) reduction of the ERG b-wave amplitude in comparison to control morphants at log0 and in comparison to WT at log-2. WT, $n=39$; control MO, $n=11$; EAAT2b MO 1, $n=39$; EAAT2b MO 2, $n=16$. $\boldsymbol{C}$, The function of EAAT2b could be demonstrated by double knockdown of both EAAT2 paralogs. Under such conditions, when glutamate uptake by Müller glia cells was impaired, we could show an even further reduction of the ERG b-wave amplitude in the double morphants in comparison to EAAT2a morphant larvae [slightly significant $(p<0.05)$ at $\log 0$, significant $(p<0.01)$ at log-1, and highly significant $(p<0.001)$ at log-3 and log-2]. EAAT2a MO 1, $n=23$; EAAT2b MO 2, $n=16$; double MO, $n=32$. $\boldsymbol{D}-\boldsymbol{F}$, Representative ERG traces of control MO injected larvae $(\boldsymbol{D})$, EAAT2a morphant ( $\boldsymbol{E}$, high dose of $\mathrm{MO} 2)$, and EAAT2b morphant (MO 2) larvae ( $\boldsymbol{F}$ ). Yellow bar represents light stimulus (starting at time 0 , ending at $100 \mathrm{~ms})$. 

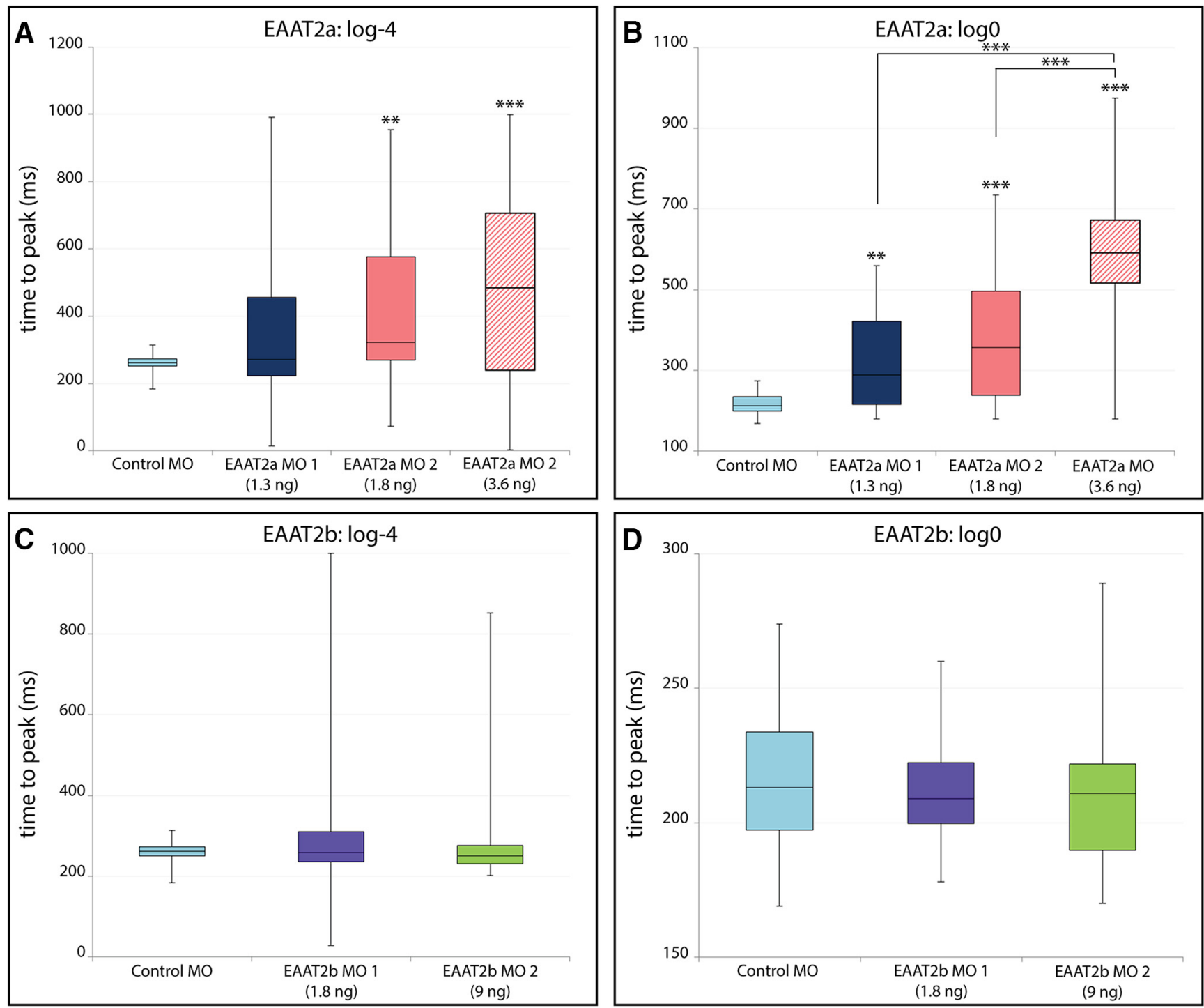

Figure 6. Time-to-peak analysis of ERG recordings. Box-and-whisker plots of time (ms) from onset of light stimulus (0 ms) to the peak of the b-wave. EAAT2a morphant larvae display changed ERG kinetics. At low light levels (A), low and high doses of EAAT2a MO 2 result in significant $(p<0.01)$ and highly significant $(p<0.001)$ increases in the time to peak, respectively. At bright light levels $(B)$, EAAT2a morphant $(\mathrm{MO} 1)$ larvae show a significant $(p<0.01)$ increase in the time to peak, whereas both levels of MO 2 result in a highly significant $(p<0.001)$ increase in the time to peak. ERG response of EAAT2b morphant fish was not decelerated in dim light conditions $(\boldsymbol{C})$ or in bright light $(\boldsymbol{D})$.

EAAT2a- and EAAT2b-injected oocytes (Fig. 7C, D, black). This outward current was more prominent for EAAT2b-expressing oocytes. Such an outward current has been described for human EAAT1 and EAAT3 and salamander sEAAT1, sEAAT2, and sEAAT5 (Eliasof and Werblin, 1993; Wadiche et al., 1995; Eliasof et al., 1998a, b) and shown to be due to the uncoupled chloride conductance that is associated with the transporter itself.

The more prominent outward current in EAAT2binjected oocytes suggested that a larger chloride conductance was associated with this paralog. To verify this hypothesis, we recorded $\mathrm{I}_{\text {Glu }}$ in a bath solution in which chloride was substituted with gluconate. Gluconate cannot permeate the chloride channel; therefore, for potentials that are positive to the chloride equilibrium potential, no current carried by an inward chloride flux should be recorded. For EAAT2a-injected oocytes, there was no difference between the glutamate current recorded in control solution and that recorded in chloride-free solution (Fig. 7C). Conversely, for EAAT2b-injected oocytes, the glutamate-induced outward current was abolished at positive potentials when recorded in a gluconate-based solution, whereas the inward current was only minimally affected (Fig. 7D). The chloride current is therefore a significant component of the total glutamate-induced current.

To estimate how much of the total $\mathrm{I}_{\text {Glu }}$ was due to the uncoupled chloride component, we calculated the charge-to-flux ratio (the charge transferred per molecule of glutamate taken up), using bath-applied tritiated gluta- 


\section{EAAT2a}

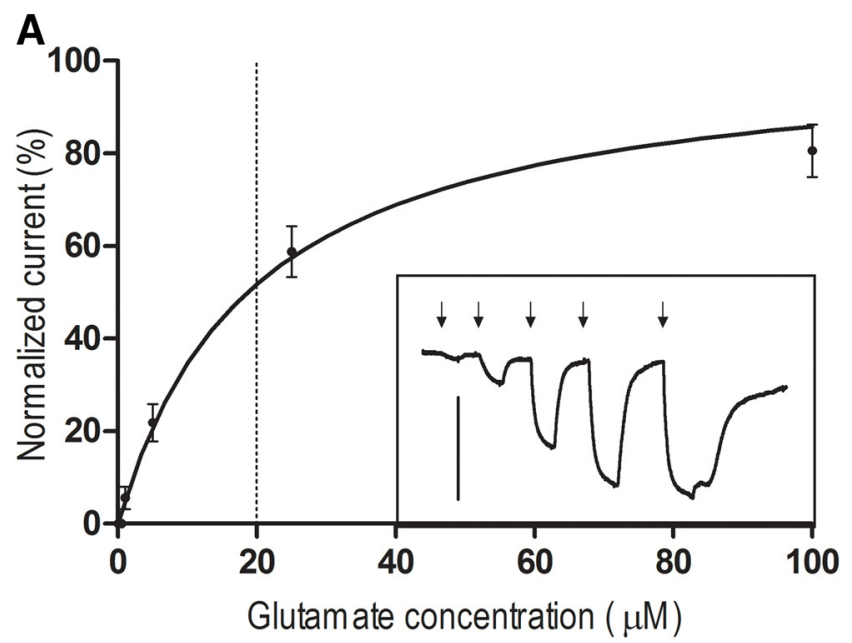

C

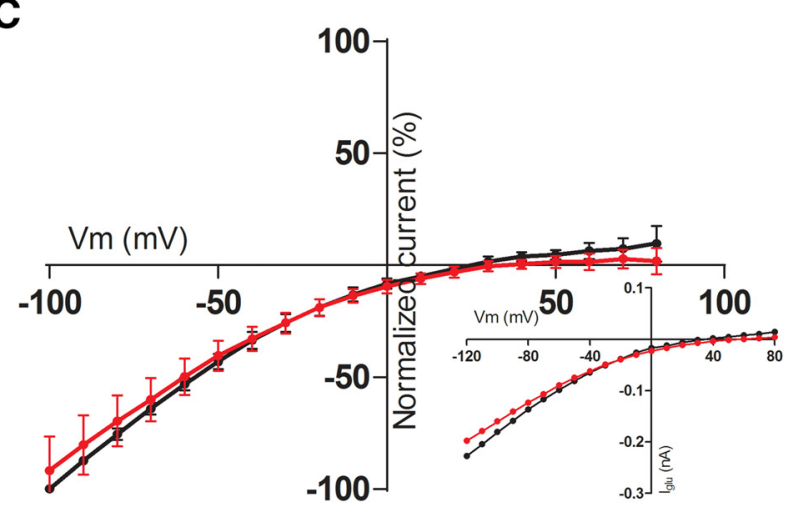

E

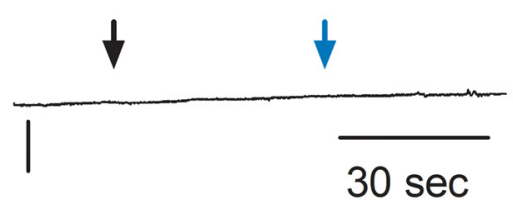

G

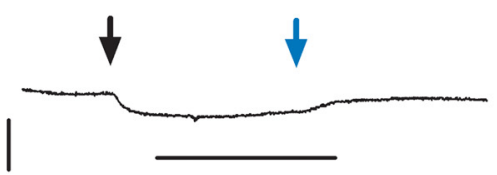

\section{EAAT2b}

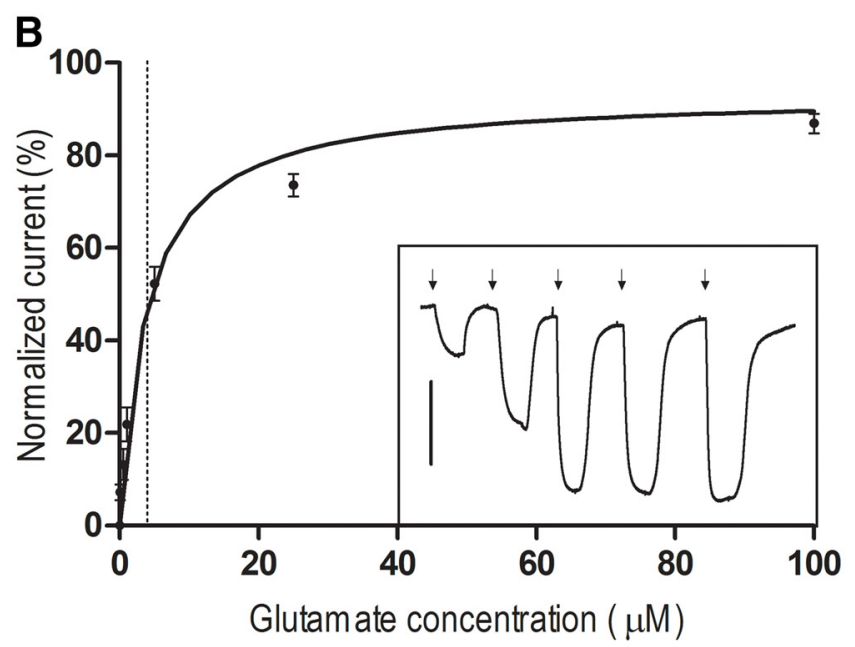

D

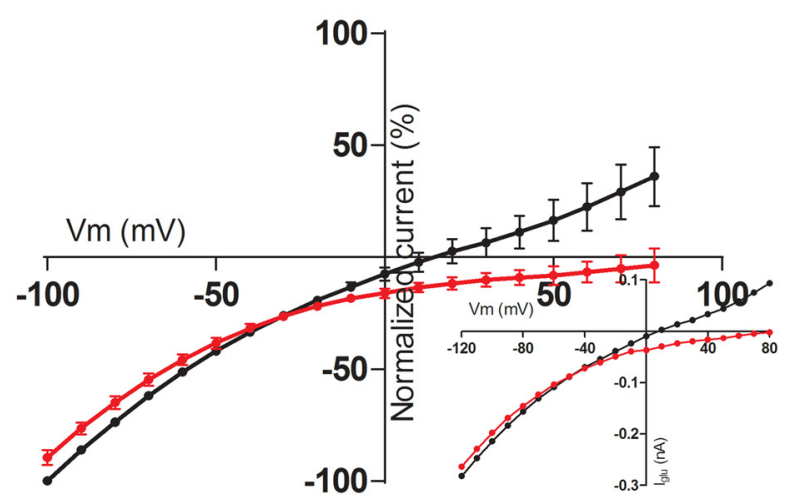

F

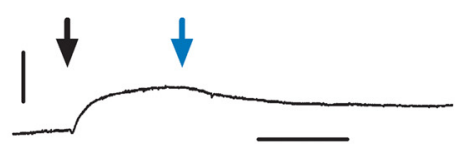

H

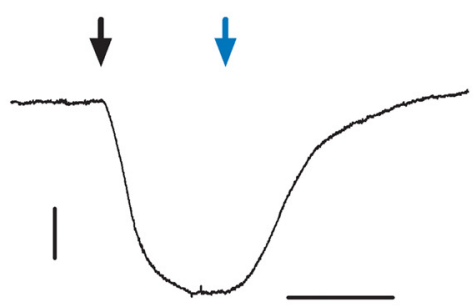

Figure 7. Two-electrode voltage clamp recordings from EAAT2a- and EAAT2b-expressing oocytes. $\boldsymbol{A}, \boldsymbol{B}$, Glutamate-evoked currents normalized to the saturating current induced by $500 \mu \mathrm{M}$ glutamate in EAAT2a-expressing $(\boldsymbol{A})$ and EAAT2b-expressing $(\boldsymbol{B})$ oocytes $(n=8)$ and fitted with the Michaelis-Menten equation. The oocytes were voltage clamped at $-50 \mathrm{mV}$. For clarity, fitted curves were 
continued

plotted only up to $100 \mu \mathrm{M}$. In the insets, inward currents were induced by increasing concentrations of L-glutamate $(1,5,25,100$, and $500 \mu \mathrm{M})$ in representative oocytes. The arrows indicate when glutamate was applied. Scale bar is 20 nA. $\boldsymbol{C}, \boldsymbol{D}$, Voltage dependence of EAAT2a-mediated (C) and EAAT2b-mediated (D) currents $(n=5)$ induced by $100 \mu \mathrm{M}$ L-glutamate in control solution (black) and a chloride-free solution (red). The data from each cell were normalized to the response elicited by $100 \mu \mathrm{M}$ L-glutamate in control solution at $-100 \mathrm{mV}$. Insets show I-V recordings from representative oocytes in normal buffer. Data under both conditions are recorded from the same cells; oocytes are from three different batches. $\boldsymbol{E}-\boldsymbol{H}$, TBOA reveals a leak current. TBOA (100 mM) was applied alone (black arrow) in control medium to EAAT2a $(\boldsymbol{E})$ and EAAT2b $(\boldsymbol{F})$ injected oocytes. In EAAT2b injected oocytes, it evoked an outward current. When oocytes were dialyzed with a chloride-free solution for $24 \mathrm{~h}$ and the control perfusing medium was exchanged with a solution containing $\mathrm{SCN}^{-}$as the main negative ion, TBOA induced an inward current in EAAT2a and EAAT2b injected oocytes $(\boldsymbol{G}, \boldsymbol{H})$. Blue arrows indicate wash from TBOA. Scale bar is $10 \mathrm{nA}$.

mate. This ratio is independent of functional expressionlevel efficiency and provides a reliable measure of the current elicited by each molecule of glutamate. At a holding potential of $-60 \mathrm{mV}$, the calculated charge-to-flux ratios were significantly different $(p<0.0008$, two-tailed $t$ test) for EAAT2a $(1.7 \pm 0.4, n=8)$ and EAAT2b $(4.5 \pm 1.7$, $n=8)$ injected oocytes.

However, at this potential, the recorded current is due to both the chloride conductance and the stoichiometrically transported ions $\left(\mathrm{Na}^{+}, \mathrm{K}^{+}\right.$, and $\left.\mathrm{H}^{+}\right)$. To confirm that the difference between the two ratios was indeed due to chloride flux, we repeated the uptake experiment at -15 $\mathrm{mV}$, the calculated chloride equilibrium potential (see Materials and Methods). The charge-to-flux ratio did not differ for the two transporters $(1.2 \pm 0.2$ for EAAT2a and $1.3 \pm 0.3$ for EAAT2b) at this potential. Therefore, we concluded that the observed difference in charge-to-flux ratio at $-60 \mathrm{mV}$ was indeed due to differences in chloride conductance. Moreover, the charge-to-flux ratio at -60 $\mathrm{mV}$ for EAAT2a was not significantly different from the uptake at $-15 \mathrm{mV}$ (ANOVA plus Tukey post hoc test), which indicated that the larger charge-to-flux ratio measured at $-60 \mathrm{mV}$ with EAAT2b-injected oocytes was mostly due to uncoupled chloride current.

\section{EAAT2a and EAAT2b differ in their leak currents}

To determine whether currents were induced only by the ligand glutamate, we checked whether DL-TBOA, a nontransportable antagonist, could block $I_{\text {Glu }}$ in EAAT2aand EAAT2b-injected oocytes. DL-TBOA blocked the glutamate-induced current as expected, but also revealed a current in EAAT2b (not in EAAT2a) injected oocytes in the absence of glutamate (Fig. 7E, F). Indeed, the antagonist blocks the channel, preventing the ions from flowing through it, and unmasks ion slippage through the transporter in the absence of substrate (glutamate in this case; Shimamoto et al., 2004).

The leak current (determined by subtracting the current in DL-TBOA from the current recorded in absence of antagonist) was inward at negative potentials and outward for potentials more positive than $-16 \mathrm{mV}$ (not shown). Leak currents have been described for most electrogenic carriers (Sonders and Amara, 1996; Ryan et al., 2004; Vandenberg et al., 2008; Andrini et al., 2008), but their function is often not clear. For some transporters, the ions contributing to the leak current can be the same ones that are translocated, whereas for others they are different. The calculated oocyte chloride equilibrium po- tential is close to the reversal potential of the EAAT2b leak current, suggesting that chloride flows through EAAT2b in the absence of glutamate. In contrast to EAAT2b, we never observed an outward current at negative potentials in the presence of DL-TBOA in EAAT2a-injected oocytes $(n=6)$.

To test whether the absence of a leak current in EAAT2a could be due to the absence of such ion slippage or to a negligible chloride conductance associated with the transporter, we recorded the DL-TBOA-induced currents in a solution in which chloride was substituted with $\mathrm{SCN}^{-}$. When DL-TBOA alone was applied to the $\mathrm{SCN}^{-}$bathing solution, an inward current was observed in all EAAT2binjected oocytes at negative potentials (Fig. $7 H$ ): in these experimental conditions, $\mathrm{SCN}^{-}$was indeed flowing into the oocyte through the EAAT2b before DL-TBOA closed the leak. Under these same conditions, even EAAT2a oocytes displayed an inward current, although small compared with the one elicited in EAAT2b-injected oocytes (Fig. 7G). This current was too small to be measured in EAAT2a-expressing oocytes bathed in standard Ringer solution, suggesting that only EAAT2b has a leak current that could exert an important physiologic role.

\section{Discussion}

The synaptic clearance of glutamate by EAATs is essential to maintain synaptic function in the CNS (Zhou and Danbolt, 2013). In this study, we focused on zebrafish EAAT2 proteins that are expressed at the photoreceptor synapse. This paralogous gene pair originated from a genome-duplication event $\sim 350$ million years ago. Intriguingly, we found the two paralogous genes and their proteins to be complementarily expressed in two distinct retinal cell types: cone photoreceptors and Müller glia cells. Although high mRNA expression levels of eaat2a are observed in the INL, only low transcript levels can be seen in photoreceptors. On the other hand, mRNA expression levels of eaat $2 b$ are high in photoreceptors and low in the INL. This weak INL expression of eaat $2 b$ and even lower transcript levels of eaat2a in photoreceptors might be a remnant of the ancestral eaat2 gene expression, which was, before the whole-genome duplication, likely expressed in both Müller glia cells and photoreceptors. Interestingly, protein expression of the corresponding eaats in these cells could not be detected using our immunofluorescence protocol. Whether this is due to the generally low protein concentration in the membrane of these cells or degradation of EAAT2b in Müller cells and 

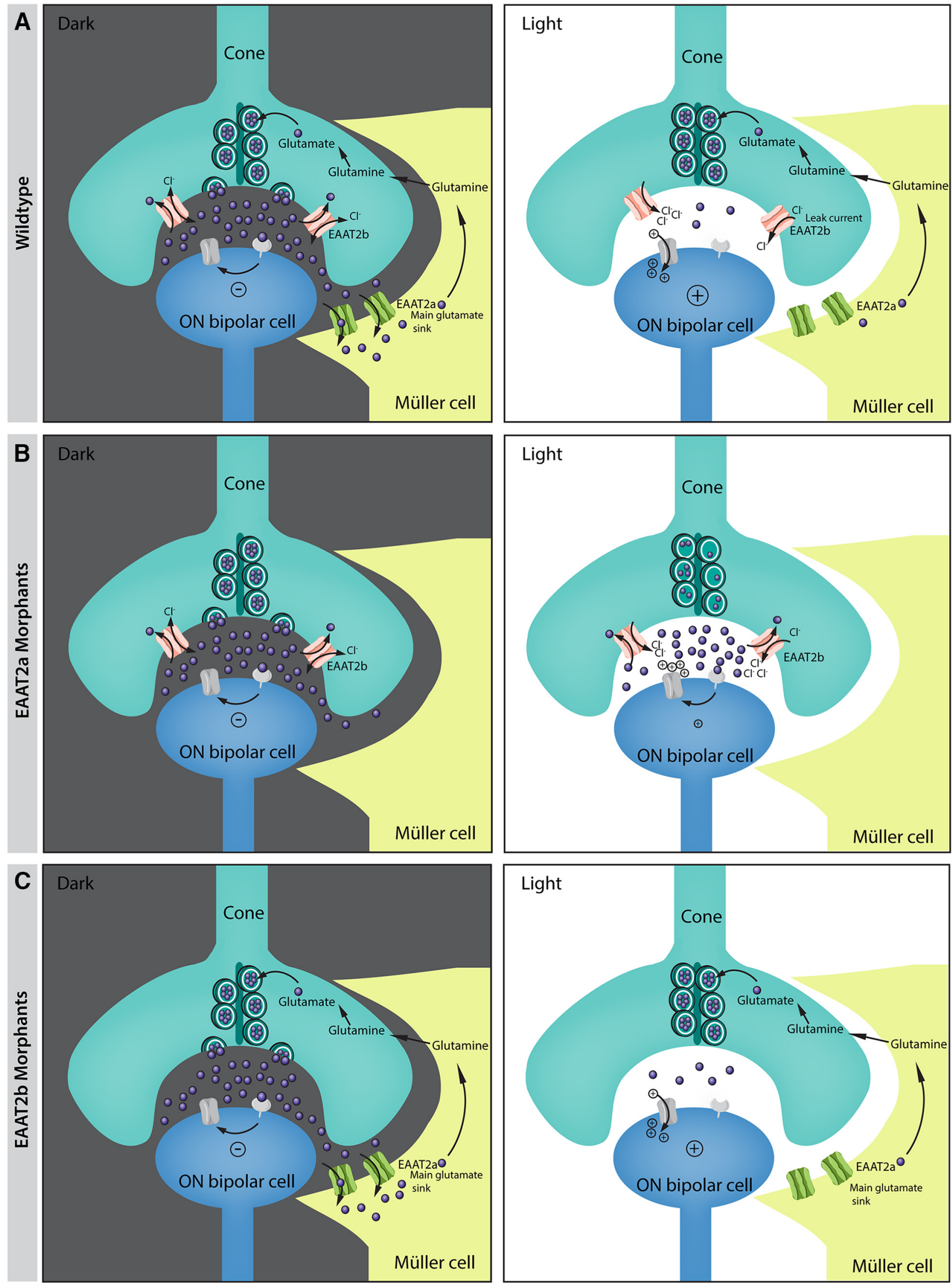

Figure 8. Schematics of photoreceptor synapse in light and dark. Illustration of changes in the photoreceptor synapse between light and dark in WT $(\boldsymbol{A})$ and EAAT2a $(\boldsymbol{B})$ and EAAT2b $(\boldsymbol{C})$ morphants. EAAT2a on Müller glia cells is responsible for the uptake of the main 
continued

load of glutamate. The uptaken glutamate in Müller cells is recycled via the glutamate-glutamine cycle. In EAAT2a morphants (B), the main load of glutamate is not being taken up and the cleft glutamate concentrations remain high, even during bright light stimuli. This may lead to binding of glutamate to postsynaptic receptors and therefore to a decreased ON-response in comparison to WT animals. The presynaptic transporter EAAT2b has a large $\mathrm{Cl}^{-}$conductance with a large leak current in absence of glutamate. During a light stimulus, when photoreceptors hyperpolarize and very few glutamate remains in the synaptic cleft, $\mathrm{Cl}^{-}$leaves the photoreceptor (leak current) and brings back the membrane potential closer to the dark resting potential. Because of the lack of such a leak current in EAAT2b morphants, cones remain in a slightly more hyperpolarized state.

EAAT2a in cones remains to be analyzed. The case of EAAT2 represents a rare case in which the subfunctionalization event led to a change not only in expression pattern but also in biophysical properties that can be directly linked to the biological function.

Unfortunately, morpholino antisense nucleotides are effective in knocking down protein only until $\sim 5 \mathrm{dpf}$. At this stage, the small size of the larval retina prevents patchclamp recordings of outer and inner retinal neurons. Furthermore, no paralog-specific pharmacologic inhibitors are available that would allow examination of the transporters in the adult retina by single-cell recordings. Therefore, functional analysis was conducted by ERG recordings. The ERG measures sum field potential changes of the retina evoked by changes in illumination. It has proven to be a robust measure of photoreceptor responses and subsequent bipolar cell activation. The ERG is a standard approach to measure outer retina function not only in model organisms, but also in the clinic (Perlman, 1995).

Knockdown of the glial transporter EAAT2a shows a highly significant reduction in the ERG b-wave, implying that glutamate levels in the synaptic cleft remain elevated even during a light stimulus, saturating postsynaptic glutamatergic receptors (Fig. $8 B$ ). This is consistent with the increased time to peak recorded for the b-wave in these animals. This delay is likely due to elevated glutamate levels in the cleft, even after light stimulation. In darkness, photoreceptors tonically release glutamate, which binds to the postsynaptic metabotropic glutamate receptor $6 \mathrm{~b}$ (Nomura et al., 1994; Huang et al., 2012). This leads, via a signaling cascade, to the closure of the cation-conducting ion channel TRPM1 (Morgans et al., 2009). During a light stimulus, photoreceptors become more hyperpolarized, which results in a decreased number of vesicles fusing at the presynapse. This, together with glutamate transporters clearing the synaptic glutamate, results in reduced concentrations of glutamate in the synaptic cleft, to an extent depending on the light stimulus intensity (Fig. 8A). In EAAT2a-depleted animals, glutamate levels do not decrease during a light stimulus to the same extent as in WT animals, implying reduced and delayed ON bipolar cell depolarization (Fig. 8B). Müller glia cell processes wrap around and coat photoreceptor synapses but do not invaginate the synapse (Burris et al., 2002). Thus released glutamate needs to diffuse from the presynaptic release site to the glial glutamate transporter EAAT2a on Müller cell processes, where it is being taken up. Such a system, with the main transporter being outside the synaptic cleft, prevents direct competition of transporter with the postsynaptic receptors and therefore keeps interference of the glutamate uptake with signal propagation to a minimum
(Gaal et al., 1998; Roska et al., 1998). In accordance with being expressed on glia cells rather than neurons, we could not associate EAAT2a with high chloride conductance or with a high leak current, which implies that its primary function is high-capacity glutamate transport and not modulation of membrane potentials.

Loss of the presynaptic glutamate transporter EAAT2b only slightly modulates the ERG b-wave, indicating that synaptic glutamate levels in knockdown animals must still be comparable to the ones in WT (Fig. 8C). The fact that the kinetics of the EAAT2b morphant ERG does not significantly differ from that in control animals further reveals that EAAT2b does not have a high glutamate turnover rate. However, EAAT2b shows a high affinity to glutamate; hence, it must either be significantly outnumbered by EAAT2a or have a lower cycling rate or capture efficiency (likelihood of glutamate being transported versus released again) than EAAT2a. Glutamate transporters have been shown to buffer glutamate and thereby shape signal transmission after a quantal event in a fast time frame, despite having slow turnover rates (Wadiche et al., 1995; Diamond and Jahr, 1997; Wadiche and Kavanaugh, 1998). Furthermore, EAAT1-3 are thought to have a capture efficiency of 0.5 , indicating that the likelihood of being released again equals the likelihood of being transported (Cavelier and Attwell, 2005; Beart and O'Shea, 2007; Tilleux and Hermans, 2007). Therefore, we assume that glutamate released by photoreceptors can bind and unbind several transporters within the cleft, before being taken up by glial transporters that surround the synapse in high numbers (Diamond and Jahr, 1997; Wadiche and Jahr, 2001; Bergles et al., 2002).

In addition, our ERG results suggest that the main vesicular glutamate pool of cones is not being replenished by presynaptic glutamate uptake via EAAT2b; otherwise EAAT2b knockdown would result in a depletion of the presynaptic vesicles, which would give us a more drastic phenotype. Glutamate vesicles of cones therefore seem to be mainly filled by glutamate recycled via the glutamate-glutamine cycle between Müller cells and photoreceptors (Fig. 8A), revealing another difference between cones and rods, which are thought to possess an almost self-sustaining glutamate uptake system (Hasegawa et al., 2006).

ERG recordings on double morphant zebrafish indicate that upon loss of both EAAT2 paralogs, glutamate uptake is further neutralized. The resulting difference in the b-wave amplitude between EAAT2a single loss and EAAT2 double knockdown is (1) the result of photoreceptors possessing a limited pool of glutamate-filled vesicles (as both the glutamate-glutamine cycle via Müller glia 
cells and the presynaptic uptake of glutamate are inhibited); (2) the result of an increased glutamate level in the synaptic cleft, even during light stimuli; or (3) both.

Binding of glutamate by EAAT2b is accompanied by the opening of chloride conductance that generates a large component of the total current. The chloride driving force and ionic flux direction depend on the chloride equilibrium potential that we suppose for zebrafish cones is between -40 and $-50 \mathrm{mV}$. These estimates stem from data obtain in goldfish ( $-50 \mathrm{mV}$; Vroman et al., 2014), salamanders (-46 mV; Thoreson and Bryson, 2004), and turtle (-47 mV; Kaneko and Tachibana, 1986) cones. As the cone dark resting potential of zebrafish is around $-40 \mathrm{mV}$ (Endeman et al., 2013), very close to the chloride equilibrium potential $\left(\mathrm{E}_{\mathrm{Cl}}\right)$, the flux of chloride that accompanies glutamate transport of EAAT2b will force the cone to return to potentials close to the dark resting potential, giving rise to previously described positive feedback (Szmajda and Devries, 2011). The presence of the large leak current that we have described for EAAT2b in the absence of glutamate will push the cone toward the resting potential, even when glutamate in the cleft is extremely low. Accordingly, a prolonged and strong light stimulus will speed up the repolarization at light off (Rowan et al., 2010), stabilizing the resting potential at depolarized potentials (Fig. $8 C$ ). The lack of such a chloride leak in EAAT2b knockdown larvae would maintain the cones in a slightly hyperpolarized status in darkness and therefore contribute to the observed slight $b$-wave reduction. This effect would become more evident in the double knockdown larvae. Indeed, in this case, the postsynaptic glutamatergic receptors would be partially saturated owing to the increased glutamate concentration in the cleft, and the hyperpolarization of the cones in darkness (and their possible loss of readily available vesicles) would further reduce the difference in cleft glutamate between dark and light conditions.

It is intriguing that the zebrafish retina acquired a system with subfunctionalized EAAT2 proteins that is comparable to the one present in the mouse retina with different EAATs. In contrast to zebrafish, mice use EAAT2 as the presynaptic transporter, whereas EAAT1 (GLAST) on Müller cells constitutes the main glutamate sink of the photoreceptor synapse (Tse et al., 2014).

\section{References}

Andrini O, Ghezzi C, Murer H, Forster IC (2008) The leak mode of type II $\mathrm{Na}(+)-\mathrm{P}(\mathrm{i})$ cotransporters. Channels (Austin, Tex.) 2:346357. Medline

Barnett NL, Pow DV (2000) Antisense knockdown of GLAST, a glial glutamate transporter, compromises retinal function. Invest Ophthalmol Vis Sci 41:585-591. Medline

Beart PM, O'Shea RD (2007) Transporters for L-glutamate: an update on their molecular pharmacology and pathological involvement. Br J Pharmacol 150:5-17. CrossRef Medline

Bergles DE, Tzingounis AV, Jahr CE (2002) Comparison of coupled and uncoupled currents during glutamate uptake by GLT-1 transporters. J Neurosci 22:10153-10162.

Branchek T (1984) The development of photoreceptors in the zebrafish, brachydanio rerio. II. Function. J Comp Neur 224:116122. CrossRef Medline
Branchek T, Bremiller R (1984) The development of photoreceptors in the zebrafish, Brachydanio rerio. I. Structure. J Comp Neur 224:107-115. CrossRef Medline

Burris C, Klug K, Ngo IT, Sterling P, Schein S (2002) How Müller glial cells in macaque fovea coat and isolate the synaptic terminals of cone photoreceptors. J Comp Neur 453:100-111. CrossRef Medline

Cavelier P, Attwell D (2005) Tonic release of glutamate by a DIDSsensitive mechanism in rat hippocampal slices. J Physiol 564:397410. CrossRef Medline

Diamond JS, Jahr CE (1997) Transporters buffer synaptically released glutamate on a submillisecond time scale. J Neurosci 17:4672-4687. Medline

Eliasof S, Arriza JL, Leighton BH, Amara SG, Kavanaugh MP (1998a) Localization and function of five glutamate transporters cloned from the salamander retina. Vis Res 38:1443-1454.

Eliasof S, Arriza JL, Leighton BH, Kavanaugh MP, Amara SG (1998b) Excitatory amino acid transporters of the salamander retina: identification, localization, and function. J Neurosci 18:698-712.

Eliasof S, Werblin F (1993) Characterization of the glutamate transporter in retinal cones of the tiger salamander. J Neurosci 13:402411. Medline

Endeman D, Klaassen LJ, Kamermans M (2013) Action spectra of zebrafish cone photoreceptors. PloS One 8:e68540. CrossRef Medline

Fairman WA, Vandenberg RJ, Arriza JL, Kavanaugh MP, Amara SG (1995) An excitatory amino-acid transporter with properties of a ligand-gated chloride channel. Nature 375:599-603. CrossRef Medline

Gaal L, Roska B, Picaud SA, Wu SM, Marc R, Werblin FS (1998) Postsynaptic response kinetics are controlled by a glutamate transporter at cone photoreceptors. J Neurophysiol 79:190-196. Medline

Gesemann M, Lesslauer A, Maurer CM, Schönthaler HB, Neuhauss SC (2010a) Phylogenetic analysis of the vertebrate excitatory/ neutral amino acid transporter (SLC1/EAAT) family reveals lineage specific subfamilies. BMC Evol Biol 10:117.

Gesemann M, Maurer CM, Neuhauss SC, Stephan CF (2010b) Excitatory amino acid transporters in the zebrafish: Letter to "Expression and functional analysis of $\mathrm{Na}(+)$-dependent glutamate transporters from zebrafish brain" from Rico et al., Brain Res Bull 83:202-206.

Glasauer SS, Neuhauss SC (2014) Whole-genome duplication in teleost fishes and its evolutionary consequences. Mol Genet Genom 289:1045-1060. CrossRef Medline

Hamaoka T, Takechi M, Chinen A, Nishiwaki Y, Kawamura S (2002) Visualization of rod photoreceptor development using GFPtransgenic zebrafish. Genesis (New York) 34:215-220.

Harada T, Harada C, Watanabe M, Inoue Y, Sakagawa T, Nakayama N, Sasaki S, Okuyama S, Watase K, Wada K, Tanaka K (1998) Functions of the two glutamate transporters GLAST and GLT-1 in the retina. Proc Natl Acad Sci U S A 95:4663-4666. Medline

Hasegawa J, Obara T, Tanaka K, Tachibana M (2006) High-density presynaptic transporters are required for glutamate removal from the first visual synapse. Neuron 50:63-74. CrossRef Medline

Huang Y-Y, Haug MF, Gesemann M, Neuhauss SC (2012) Novel expression patterns of metabotropic glutamate receptor 6 in the zebrafish nervous system. PloS One 7:e35256. CrossRef Medline

Kaneko A, Tachibana M (1986) Effects of gamma-aminobutyric acid on isolated cone photoreceptors of the turtle retina. J Physiol 373:443-461. Medline

Larison KD, Bremiller R (1990) Early onset of phenotype and cell patterning in the embryonic zebrafish retina. Development (Cambridge, England) 109:567-576.

Levinger E, Zemel E, Perlman I (2012) The effects of excitatory amino acids and their transporters on function and structure of the distal retina in albino rabbits. Doc Ophthalmol 125:249-265. CrossRef Medline

Levy LM, Warr O, Attwell D (1998) Stoichiometry of the glial glutamate transporter GLT-1 expressed inducibly in a Chinese hamster 
ovary cell line selected for low endogenous $\mathrm{Na}+$-dependent glutamate uptake. J Neurosci 18:9620-9628.

Makhankov YV, Rinner O, Neuhauss SC (2004) An inexpensive device for non-invasive electroretinography in small aquatic vertebrates. J Neurosci Methods 135:205-210. CrossRef Medline

McKeown KA, Moreno R, Hall VL, Ribera AB, Downes GB (2012) Disruption of Eaat2b, a glutamate transporter, results in abnormal motor behaviors in developing zebrafish. Dev Biol 362:162-171. CrossRef Medline

Morgans CW (2000) Neurotransmitter release at ribbon synapses in the retina. Immunol Cell Biol 78:442-446. CrossRef Medline

Morgans CW, Zhang J, Jeffrey BG, Nelson SM, Burke NS, Duvoisin $R M$, Brown RL (2009) TRPM1 is required for the depolarizing light response in retinal ON-bipolar cells. Proc Natl Acad Sci U S A 106:19174-19178. CrossRef Medline

Mullins MC, Hammerschmidt $M$, Haffter $P$, Nüsslein-Volhard $C$ (1994) Large-scale mutagenesis in the zebrafish: in search of genes controlling development in a vertebrate. Curr Biol 4:189202. CrossRef

Nomura A, Shigemoto R, Nakamura Y, Okamoto N, Mizuno N, Nakanishi S (1994) Developmentally regulated postsynaptic localization of a metabotropic glutamate receptor in rat rod bipolar cells. Cell 77:361-369. Medline

Owe SG, Marcaggi P, Attwell D (2006) The ionic stoichiometry of the GLAST glutamate transporter in salamander retinal glia. J Physiol 577:591-599. CrossRef Medline

Palmer MJ, Taschenberger H, Hull C, Tremere L, von Gersdorff H (2003) Synaptic activation of presynaptic glutamate transporter currents in nerve terminals. J Neurosci 23:4831-4841.

Perlman I (1995) The Electroretinogram: ERG. In: Webvision: The Organization of the Retina and Visual System (Kolb H, Fernandez E, Nelson R, eds). University of Utah, Salt Lake City, UT.

Picaud SA, Larsson HP, Grant GB, Lecar H, Werblin FS (1995) Glutamate-gated chloride channel with glutamate-transporter-like properties in cone photoreceptors of the tiger salamander. J Neurophysiol 74:1760-1771. Medline

Rauen T (2000) Diversity of glutamate transporter expression and function in the mammalian retina. Amino Acids 19:53-62. Medline

Rauen T, Taylor WR, Kuhlbrodt K, Wiessner M (1998) High-affinity glutamate transporters in the rat retina: a major role of the glial glutamate transporter GLAST-1 in transmitter clearance. Cell Tissue Res 291:19-31. CrossRef

Roska B, Gaal L, Werblin FS (1998) Voltage-dependent uptake is a major determinant of glutamate concentration at the cone synapse: an analytical study. J Neurophysiol 80:1951-1960. Medline

Rowan MJ, Ripps H, Shen W (2010) Fast glutamate uptake via EAAT2 shapes the cone-mediated light offset response in bipolar cells. J Physiol 588:3943-3956. CrossRef Medline

Ryan RM, Mitrovic AD, Vandenberg RJ (2004) The chloride permeation pathway of a glutamate transporter and its proximity to the glutamate translocation pathway. J Biol Chem 279:20742-20751. CrossRef Medline

Schmitz F (2009) The making of synaptic ribbons: how they are built and what they do. Neurosci 15:611-624. CrossRef
Shimamoto K, Sakai R, Takaoka K, Yumoto N, Nakajima T, Amara SG, Shigeri Y (2004) Characterization of novel L-threo-betabenzyloxyaspartate derivatives, potent blockers of the glutamate transporters. Mol Pharmacol 65:1008-1015. CrossRef Medline

Sonders MS, Amara SG (1996) Channels in transporters. Curr Opin Neurobiol 6:294-302. Medline

Szmajda BA, Devries SH (2011) Glutamate spillover between mammalian cone photoreceptors. J Neurosci 31:13431-13441. CrossRef

Takechi M, Hamaoka T, Kawamura S (2003) Fluorescence visualization of ultraviolet-sensitive cone photoreceptor development in living zebrafish. FEBS Lett 553:90-94. Medline

Takechi M, Seno S, Kawamura S (2008) Identification of cis-acting elements repressing blue opsin expression in zebrafish UV cones and pineal cells. J Biol Chem 283:31625-31632. CrossRef Medline

Thoreson WB, Bryson EJ (2004) Chloride equilibrium potential in salamander cones. BMC Neurosci 5:53. CrossRef Medline

Tilleux S, Hermans E (2007) Neuroinflammation and regulation of glial glutamate uptake in neurological disorders. J Neurosci Res 85: 2059-2070. CrossRef Medline

Tse DY, Chung I, Wu SM (2014) Pharmacological inhibitions of glutamate transporters EAAT1 and EAAT2 compromise glutamate transport in photoreceptor to ON-bipolar cell synapses. Vis Res 103:49-62. CrossRef Medline

Vandenberg RJ, Huang S, Ryan RM (2008) Slips, leaks and channels in glutamate transporters. Channels (Austin) 2:51-58. CrossRef

Vandenbranden CA, Verweij J, Kamermans M, Müller LJ, Ruijter JM, Vrensen GF, Spekreijse H (1996) Clearance of neurotransmitter from the cone synaptic cleft in goldfish retina. Vis Res 36:38593874. Medline

Veruki ML, Mørkve SH, Hartveit E (2006) Activation of a presynaptic glutamate transporter regulates synaptic transmission through electrical signaling. Nat Neurosci 9:1388-1396. CrossRef Medline

Vroman R, Klaassen LJ, Howlett MHC, Cenedese V, Klooster J, Sjoerdsma T, Kamermans M (2014) Extracellular ATP hydrolysis inhibits synaptic transmission by increasing $\mathrm{pH}$ buffering in the synaptic cleft. PLoS Biol 12:e1001864. CrossRef

Wadiche JI, Amara SG, Kavanaugh MP (1995) Ion fluxes associated with excitatory amino acid transport. Neuron 15:721-728. Medline

Wadiche JI, Jahr CE (2001) Multivesicular release at climbing fiberPurkinje cell synapses. Neuron 32:301-313. Medline

Wadiche JI, Kavanaugh MP (1998) Macroscopic and microscopic properties of a cloned glutamate transporter/chloride channel. J Neurosci 18:7650-7661. Medline

Wersinger E, Schwab Y, Sahel J-A, Rendon A, Pow DV, Picaud S, Roux MJ (2006) The glutamate transporter EAAT5 works as a presynaptic receptor in mouse rod bipolar cells. J Physiol 577: 221-234. CrossRef Medline

White RD, Neal MJ (1976) The uptake of L-glutamate by the retina. Brain Res 111:79-93. Medline

Zerangue N, Kavanaugh MP (1996) Flux coupling in a neuronal glutamate transporter. Nature 383:634-637. CrossRef Medline

Zhou Y, Danbolt NC (2013) GABA and glutamate transporters in brain. Front Endocrinol 4:165. CrossRef Medline 